\title{
Recursive State Estimation for Stochastic Complex Networks under Round-Robin Communication Protocol: Handling Packet Disorders
}

\author{
Dan Liu, Zidong Wang, Yurong Liu, Fawaz E. Alsaadi, and Fuad E. Alsaadi
}

\begin{abstract}
This paper investigates the recursive state estimation problem for a class of discrete-time stochastic complex networks with packet disorders under Round-Robin (RR) communication protocols. The phenomenon of packet disorders results from the random transmission delays during the signal propagation process due to the unpredictable fluctuations of the network load, and such random delays are modeled by a set of random variables satisfying certain known probability distributions. For the sake of lessening the communication burden and abating the data collisions, the RR protocol is introduced to govern the order of the nodes for data transmission. Under the scheduling of the RR protocol, only one node is allowed to gain the access to the network at each time instant. Then, a recursive estimator is devised to guarantee an upper bound for the estimation error covariance, and then the obtained upper bound is locally minimized by adequately choosing the estimator parameters. Furthermore, the boundedness of estimation error is analyzed in the sense of mean square with the help of stochastic analysis techniques. At last, a simulation example is presented to show the applicability of the proposed estimator design scheme.
\end{abstract}

Index Terms-Recursive state estimation, complex networks, packet disorders, Round-Robin protocol, mean-square boundedness.

\section{INTRODUCTION}

A complex network $(\mathrm{CN})$ is comprised of a mass of nodes that are distributed and connected according to certain topologies, where each node represents an individual with dynamical behavior. CNs are well known to be an abstraction of many practical networks including social networks, biological networks and electrical power grids, and have therefore attracted an ever-increasing research interest in the past few decades

This project was funded by the Deanship of Scientific Research (DSR) at King Abdulaziz University, Jeddah, Saudi Arabia, under grant no. (RG19-611-42). This work was also supported by National Natural Science Foundation of China under Grants 61773017, 61873148, 61873230 and 61933007, the Royal Society of the UK, and the Alexander von Humboldt Foundation of Germany. (Corresponding author: Yurong Liu.)

D. Liu is with the Department of Mathematics, Yangzhou University, Yangzhou 225002, China.

Z. Wang is with the Department of Computer Science, Brunel University London, Uxbridge, Middlesex, UB8 3PH, United Kingdom (Email: Zidong. Wang@brunel.ac.uk).

Y. Liu is with the Department of Mathematics, Yangzhou University, Yangzhou 225002, China, and also with the School of Mathematics and Physics, Yancheng Institute of Technology, Yancheng 224051, China (Email: yrliu@yzu.edu.cn).

Fawaz E. Alsaadi is with the Department of Information Technology, Faculty of Computing and Information Technology, King Abdulaziz University, Jeddah, Saudi Arabia.

Fuad E. Alsaadi is with the Department of Electrical and Computer Engineering, Faculty of Engineering, King Abdulaziz University, Jeddah, Saudi Arabia. simply because of their engineering significance, see e.g. [1] [4], [8], [9], [43] for some representative results. In engineering practice, an inevitable phenomenon that the CNs would suffer from is the so-called stochastic disturbance which, in turn, would have direct influence on the dynamical behaviors of the CNs themselves. As such, it is of great importance to take the stochastic disturbance into consideration when analyzing the dynamics of the $\mathrm{CN}$ and, along this direction, much research has been done in [20], [29], [35], [40]. For instance, the global synchronization problem has been studied in [35] for the CNs in the presence of randomly occurred nonlinearities, mixed time-delays and stochastic noises. Furthermore, in [29], a new $H_{\infty}$ synchronization problem has been discussed for a class of discrete time-varying stochastic complex networks (SCNs) over a finite horizon.

In the practical applications, the network states of $\mathrm{CNs}$ are vitally important as they represent physical quantities that need to be closely monitored and accurately measured. Unfortunately, exact knowledge of the network states is often unavailable because of inherent resource limits in the context of large scales of the nodes and complicated topology of the networks. Therefore, it becomes an imperative task to estimate the network states of the CNs through attainable network measurements that might be incomplete, sparse and noisy. To date, considerable effort has been dedicated to the state estimation (SE) problem and numerous results have been reported in the literature, see e.g. [10], [11], [16], [47]. In particular, the recursive $\mathrm{SE}$ issue has been investigated in [13] for the SCNs with missing measurements, where the estimator gain can be obtained recursively at each time instant for each node by solving certain recursive matrix equations. Subsequently, a recursive estimator has been presented in [12] for a class of time-varying SCNs with uncertain inner coupling and quantized measurements. In addition, a partialnodes-based approach has been developed in [25] to design the estimator for discrete-time delayed SCNs.

In a typical communication network with limited bandwidth, it is not uncommon that the signal transmission undergoes the so-called packet disorder, which means that the data packet sent earlier (later) might reach its destination later (earlier), and this violates the "first send first arrive" principle. Obviously, the phenomenon of packet disorders (PPD) results from the random transmission delays during the signal propagation process due to the unpredictable fluctuation of the network load. In this case, the latest arrival packet might contain outdated system information which, if not properly dealt with, 
would lead to the performance degradation of the designed estimator. Unfortunately, comparing to other network-induced phenomena (e.g. packet dropout and signal quantization), the PPD has received far less research attention due probably to the difficulty in characterizing the random nature of the packet arrivals.

So far, in the literature of networked control systems (NC$\mathrm{Ss}$ ), there have been a few initial research results concerning the phenomenon of packet disorders, see e.g. [6], [14], [18], [19], [33], [41], [42], [48], where the PPD has been assumed to take place in the communication channel from the sensor to controller/filter. Nevertheless, the PPD could also occur during the sensing process where the measurement outputs experience random transmission delays before their arrivals at the ultimate receivers, see [34] and the references therein. To our knowledge, the PPD issue with respect to random sensor delays (RSDs) has not gained much research interest yet, and the first motivation of this paper is therefore to address the recursive SE problem for SCNs with RSD-induced packet disorders.

Despite the steady revolution of the wireless communication technology, the limited bandwidth remains as a main concern that would lead to data collisions when multiple data packets are transmitted simultaneously through a shared network [5], [22], [27], [39], [44]. A popular way of better utilizing the limited network resource is to adopt the communication protocols as so to administer the transmission order of network nodes, and some frequently deployed protocols include the Round-Robin (RR) protocol [17], [23], [24], [26], [37], [38], [46], the stochastic communication protocol [7], [15], [31], [45] and the Try-Once-Discard (TOD) protocol [30], [32]. Among others, the RR protocol stands out for its simple periodicity and easy implementation, under which only one node has privilege to enter the network at each time instant according to a fixed circle order. Over the past few years, although some preliminary results have appeared under RR protocols, the design of RR-regulated recursive estimator has not aroused adequate research attention yet, let alone the case where the PPD is also involved. To this end, the second motivation of this paper is to examine the impact from the $\mathrm{RR}$ protocol on the corresponding estimation performance.

In this article, we endeavor to address the recursive SE problem for a class of SCNs with packet disorders. A RR protocol is utilized to equally allocate the network resources to the nodes by only allowing one node to gain the access to the network at each time instant in a circular order, thereby mitigating the data congestion. In the presence of both packet disorders and RR protocols, an upper bound of estimation error covariance is derived and subsequently minimized via appropriate design of estimator gain. By resorting to the stochastic analysis techniques, we also analyze the mean-square boundedness of the error dynamics of the state estimation. In doing so, we are facing the following substantial difficulties: 1) how to describe the RSDs properly; 2) how to characterize the updating rule of the RR protocols; 3) how to design an appropriate estimator that mitigates the effect of the RSDs and also reflects the influence of the RR protocols; and 4) how to guarantee the boundedness of estimation errors.
To deal with the above difficulties, the primary contributions of this article are highlighted as follows: 1) the recursive SE issue is, for the first time, investigated for SCNs subject to packet disorders and RR protocol; 2) the considered RSDs resulting in PPD are described by a sequence of random variables following a known probability distribution; 3) a new measurement model is proposed to account for the RSDs and RR protocols and then a novel recursive estimator is developed by using an integer-valued function; and 4) a sufficient condition is derived to guarantee that the estimation error is exponentially bounded in the mean-square sense.

Notation: The notation used here is fairly standard. For a matrix $Z, Z^{T}, Z^{-1}$ and $\operatorname{tr}\{Z\}$ represent, respectively, the transpose, the inverse and the trace of matrix $Z$. $\operatorname{col}\{\ldots\}$ denotes a column vector composed of elements and $\operatorname{diag}\{\ldots\}$ represents a block-diagonal matrix. The notation $\|\cdot\|$ denotes the Euclidean (spectral) norm of real vectors (matrices). $\mathbb{E}\{\cdot\}$ stands for the expectation operator. The Kronecker product of a $r \times s$ matrix $M$ and a $t \times h$ matrix $N$ is defined by a $r t \times s h$ matrix $M \otimes N$ as follows:

$$
M \otimes N=\left[\begin{array}{ccc}
m_{11} N & \cdots & m_{1 s} N \\
\vdots & & \vdots \\
m_{r 1} N & \cdots & m_{r s} N
\end{array}\right] .
$$

The Kronecker delta function $\delta(a)$ is defined by $\delta(a)=1$ if $a=0$ and $\delta(a)=0$ otherwise. $\bmod (p, q)$ denotes the remainder of the integer $p$ divided by the positive integer $q$. The floor function $\lfloor b\rfloor$ equals the largest integer no larger than $b$ with $b$ being an arbitrary real number.

\section{Problem Formulation}

Consider a class of discrete-time SCNs consisting of $M$ coupled nodes:

$$
\begin{aligned}
x_{i}(r+1)= & A_{i}(r) x_{i}(r)+f\left(x_{i}(r)\right)+\sum_{j=1}^{M} \omega_{i j} \Gamma x_{j}(r) \\
& +B_{i}(r) w(r), \\
\vec{y}_{i}(r)= & C_{i}(r) x_{i}\left(r-\tau_{i}(r)\right)+D_{i}(r) v(r),
\end{aligned}
$$

where $x_{i}(r) \in \mathbb{R}^{m}$ and $\vec{y}_{i}(r) \in \mathbb{R}^{n}$ are the system state and the measurement output of the $i$-th $(i=1,2, \ldots, M)$ node, respectively; $f(\cdot)$ is a known nonlinear function which is continuously differentiable; $w(r) \in \mathbb{R}^{w}$ and $v(r) \in \mathbb{R}^{v}$ are, respectively, zero-mean white sequences distributed in bounded domains with covariances $Q(r)>0$ and $R(r)>0$; $A_{i}(r), B_{i}(r), C_{i}(r)$ and $D_{i}(r)$ are known matrices; $\Gamma=$ $\operatorname{diag}\left\{\gamma_{1}, \gamma_{2}, \ldots, \gamma_{m}\right\} \geq 0$ is an inner-coupling matrix, where $\gamma_{j} \neq 0$ stands for the linking with $j$-th state variable; $W=\left[\omega_{i j}\right]_{M \times M}$ is the coupled configuration matrix with $\omega_{i j}>0$ if node $i$ can receive the information from node $j$, otherwise $\omega_{i j}=0$, which satisfies $W=W^{T}$ and

$$
\sum_{j=1}^{M} \omega_{i j}=\sum_{j=1}^{M} \omega_{j i}=0 ;
$$

$\tau_{i}(r)$ denotes the RSD at the time instant $r$ that obeys the following statistical distribution law:

$$
\operatorname{Prob}\left\{\tau_{i}(r)=s\right\}=p_{s}, \quad s=0,1, \ldots, q,
$$


where $q$ is a known positive integer, $p_{s}(s=0,1, \ldots, q)$ are known constants satisfying $0 \leq p_{s} \leq 1$ and $\sum_{s=0}^{q} p_{s}=1$. We assume that $x_{i}(r)=\varphi_{i}(r)(r=-q,-q+1, \ldots, 0)$ where $\varphi_{i}(r)$ is the initial condition.

Remark 1: As shown in (1b), the model of measurement output $\vec{y}_{i}(r)$ contains the term $x_{i}\left(r-\tau_{i}(r)\right)$ that is related to RSDs occurring in the sensing process, and such RSDs would result in packet dropouts. In most existing literature, it has been assumed that the random delays follow a Markov chain satisfying a precisely known transition probability matrix [18]. Recognizing the difficulty of identifying such a transition probability matrix, in this paper, we use a set of random variables (with known probability distribution) to characterize the random delays [21]. On the other hand, as opposed to those occurring in the sensor-to-filter channel in the literature, the random delays in this paper take place in the sensing process and appear in the measurement outputs, which are more likely to induce the packet disorders and bring in more difficulties in the subsequent analysis.

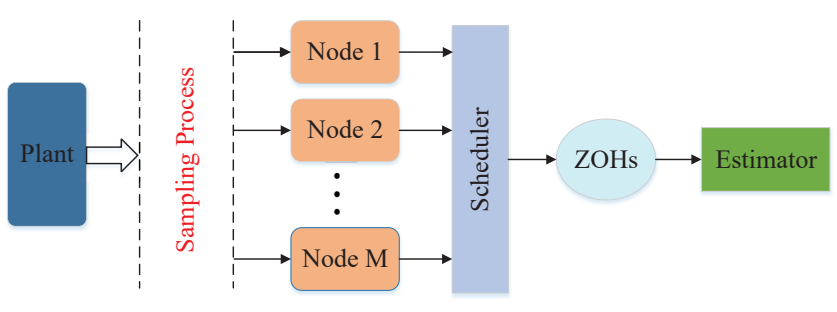

Fig. 1. Diagrammatic sketch of estimation system with a RR protocol.

For notation simplicity, we let

$$
\begin{aligned}
x(r) & \triangleq \operatorname{col}\left\{x_{1}(r), x_{2}(r), \ldots, x_{M}(r)\right\}, \\
\vec{y}(r) & \triangleq \operatorname{col}\left\{\vec{y}_{1}(r), \vec{y}_{2}(r), \ldots, \vec{y}_{M}(r)\right\}, \\
A(r) & \triangleq \operatorname{diag}\left\{A_{1}(r), A_{2}(r), \ldots, A_{M}(r)\right\}, \\
B(r) & \triangleq \operatorname{col}\left\{B_{1}(r), B_{2}(r), \ldots, B_{M}(r)\right\}, \\
f(x(r)) & \triangleq \operatorname{col}\left\{f\left(x_{1}(r)\right), f\left(x_{2}(r)\right), \ldots, f\left(x_{M}(r)\right)\right\}, \\
\bar{C}_{i}(r) & \triangleq \operatorname{diag}\{\underbrace{0_{n \times m}, \ldots, 0_{n \times m}}_{i-1}, C_{i}(r), \underbrace{0_{n \times m}, \ldots, 0_{n \times m}}_{M-i}\}, \\
D(r) & \triangleq \operatorname{col}\left\{D_{1}(r), D_{2}(r), \ldots, D_{M}(r)\right\} .
\end{aligned}
$$

By utilizing the Kronecker product, we rewrite the SCNs (1a)-(1b) as:

$$
x(r+1)=[A(r)+(W \otimes \Gamma)] x(r)+f(x(r))+B(r) w(r),
$$

$$
\vec{y}(r)=\sum_{i=1}^{M} \bar{C}_{i}(r) x\left(r-\tau_{i}(r)\right)+D(r) v(r)
$$

with the mean and covariance matrix of the initial condition $x(0)$ being $\bar{x}(0)$ and $P(0)$, respectively.

In this paper, the measurement outputs sent by the sensors are transmitted to a remote estimator through a constrained communication network, and this might give rise to data collisions when multiple nodes transmit signals simultaneously.
To avert excessive data collisions and improve the utilization efficiency of the communication resource, the RR protocol is used to regulate the transmission order of the measurement signal, where the corresponding schematic sketch is shown in Fig. 1. Under the scheduling of the RR protocol, only one node is permitted to send its data at each time instant according to a circular order. Let $y(r) \triangleq \operatorname{col}\left\{y_{1}(r), y_{2}(r), \ldots, y_{M}(r)\right\}$ denote the actual measurement received by the estimator. According to the RR protocol, it follows that

$$
y_{i}(r)= \begin{cases}\vec{y}_{i}(r), & \text { if } \bmod (r-1, M)=0, \\ y_{i}(r-1), & \text { otherwise. }\end{cases}
$$

For the sake of convenience, we assume that $y(r)=0$ for $r<0$ and define $\Phi_{i} \triangleq \operatorname{diag}\{\delta(i-1) I, \delta(i-2) I, \ldots, \delta(i-$ $M) I\}(i=1,2, \ldots, M)$. Then, it can be inferred that

$$
y(r)=\sum_{l=0}^{M-1} \Phi_{\sigma(r-l)} \vec{y}(r-l),
$$

where

$$
\sigma(r)= \begin{cases}M, & r=0, \\ \bmod (r-1, M)+1, & \text { otherwise }\end{cases}
$$

represents the selected node having the privilege to transmit its measurement signal at time instant $r$. Moreover, we set $\sigma(r)=$ $r+M$ and $\vec{y}(r)=\vec{y}(0)$ for $r=-M+1,-M+2, \ldots, 0$ with $\vec{y}(0)$ being the initial measurement.

Remark 2: The RR protocol is utilized in this paper to schedule the data transmission so as to mitigate the communication burden. Traditionally, the updating rule of the RR protocol is given by (3) as in [46]. In this paper, such a rule has been equivalently presented as (4), with which the system state and estimator input do not need to be augmented, thereby facilitating the subsequent mathematical derivation.

Based on the scheduled measurement (4), a recursive estimator is constructed as follows:

$$
\begin{aligned}
\hat{x}(r+1 \mid r)= & {[A(r)+(W \otimes \Gamma)] \hat{x}(r \mid r)+f(\hat{x}(r \mid r)), } \\
\hat{x}(r+1 \mid r+1)= & \hat{x}(r+1 \mid r)+G(r+1)[y(r+1) \\
& -\sum_{l=0}^{M-1} \sum_{i=1}^{M} \Phi_{\sigma(r-l+1)} \bar{C}_{i}(r-l+1) \\
& \times \hat{x}(r-l+1-d \mid r-l-d)]
\end{aligned}
$$

where $\hat{x}(r \mid r) \in \mathbb{R}^{m}$ represents the estimate of $x(r)$ with $\hat{x}(0 \mid 0)=\bar{x}(0) ; \hat{x}(r+1 \mid r) \in \mathbb{R}^{m}$ stands for the one-step prediction of $x(r+1)$ at time instant $r ; G(r+1)$ is the estimator parameter; $d$ is an integer which satisfies

$$
d= \begin{cases}\lfloor\bar{\tau}\rfloor, & \text { if } \bar{\tau}-\lfloor\bar{\tau}\rfloor<\frac{1}{2}, \\ \lfloor\bar{\tau}\rfloor+1, & \text { otherwise }\end{cases}
$$

with $\bar{\tau} \triangleq \mathbb{E}\left\{\tau_{i}(r-l+1)\right\}=\sum_{s=0}^{q} s p_{s}(i=1,2, \ldots, M)$ and $\lfloor\bar{\tau}\rfloor$ representing the biggest integer no bigger than $\bar{\tau}$. 
The main purpose of this paper is to find an upper bound matrix $\Theta(r+1 \mid r+1)$ for the estimation error covariance

$\mathbb{E}\left\{[x(r+1)-\hat{x}(r+1 \mid r+1)][x(r+1)-\hat{x}(r+1 \mid r+1)]^{T}\right\}$

on the basis of the proposed estimator (5a)-(5b). Furthermore, the upper bound $\Theta(r+1 \mid r+1)$ would be minimized at each time instant by properly choosing the estimator parameter.

Remark 3: In the proposed estimator (5a)-(5b), there are tight couplings between the nodes, the RR protocols, as well as the packet disorders. To be specific, the innovation structure

$y(r+1)-\sum_{l=0}^{M-1} \sum_{i=1}^{M} \Phi_{\sigma(r-l+1)} \bar{C}_{i}(r-l+1) \hat{x}(r-l+1-d \mid r-l-d)$

reflects, to some extent, the influence from the RR protocols and the packet disorders. The integer $d$, which appears in the innovation structure, is closely related to the random delay $\tau_{i}(r)$. Generally speaking, a reasonable estimator should not involve any random variables (e.g. $\left.\tau_{i}(r)\right)$ for its implementability. For this reason, the mathematical expectation $\bar{\tau}$ of $\tau_{i}(r)$ is taken here. It should be mentioned that, $\bar{\tau}$ might not be an integer, which means that $\bar{\tau}$ is unsuitable for representing the time instant in the discrete-time setting, and thus $\bar{\tau}$ is rounded to $d$. As such, the estimator proposed in this paper is capable of resisting the impacts from the packet disorders.

\section{MAin Results}

In this section, an upper bound is first derived for the estimation error covariance, and is then locally minimized by properly selecting the estimator parameter.

First of all, the one-step prediction error denoted by

$$
e(r+1 \mid r) \triangleq x(r+1)-\hat{x}(r+1 \mid r),
$$

is calculated from (2a) and (5a) as

$$
\begin{aligned}
e(r+1 \mid r)= & {[A(r)+(W \otimes \Gamma)] e(r \mid r)+f(x(r)) } \\
& -f(\hat{x}(r \mid r))+B(r) w(r) .
\end{aligned}
$$

Expanding $f(x(r))$ in a Taylor series around $\hat{x}(r \mid r)$, we obtain that

$$
f(x(r))=f(\hat{x}(r \mid r))+F(r) e(r \mid r)+U(r) S(r) T(r) e(r \mid r),
$$

where

$$
\left.F(r) \triangleq \frac{\partial f(x)}{\partial x}\right|_{x=\hat{x}(r \mid r)},
$$

and the term $U(r) S(r) T(r) e(r \mid r)$ stands for the linearization error with $U(r)$ and $T(r)$ being the known matrices, and $S(r)$ being an unknown matrix that satisfies $S(r) S^{T}(r) \leq I$. Furthermore, the prediction error can be further rewritten as

$$
\begin{aligned}
e(r+1 \mid r)= & {[A(r)+(W \otimes \Gamma)+F(r)+U(r) S(r) T(r)] } \\
& \times e(r \mid r)+B(r) w(r) .
\end{aligned}
$$

Denoting the estimation error as

$$
e(r+1 \mid r+1) \triangleq x(r+1)-\hat{x}(r+1 \mid r+1),
$$

we subtract (5b) from (2a) to have

$$
e(r+1 \mid r+1)
$$

$$
\begin{aligned}
& =e(r+1 \mid r)-G(r+1)\left[\sum_{l=0}^{M-1} \sum_{i=1}^{M} \Phi_{\sigma(r-l+1)}\right. \\
& \quad \times \bar{C}_{i}(r-l+1) x\left(r-l+1-\tau_{i}(r-l+1)\right) \\
& +\sum_{l=0}^{M} \Phi_{\sigma(r-l+1)} D(r-l+1) v(r-l+1) \\
& \quad-\sum_{l=0}^{M-1} \sum_{i=1}^{M} \Phi_{\sigma(r-l+1)} \bar{C}_{i}(r-l+1) \\
& \quad \times \hat{x}(r-l+1-d \mid r-l-d)]
\end{aligned}
$$

Adding the zero term $G(r+1) \sum_{i=1}^{M} \bar{C}_{i}(r+1) e(r+1 \mid r)-$ $G(r+1) \sum_{i=1}^{M} \bar{C}_{i}(r+1) e(r+1 \mid r)$ to the right-hand side of (9), we have

$$
\begin{aligned}
& \quad e(r+1 \mid r+1) \\
& =\left(I-G(r+1) \sum_{i=1}^{M} \bar{C}_{i}(r+1)\right) e(r+1 \mid r) \\
& +G(r+1) \sum_{i=1}^{M} \bar{C}_{i}(r+1) e(r+1 \mid r) \\
& \quad-G(r+1) \sum_{l=0}^{M-1} \sum_{i=1}^{M} \Phi_{\sigma(r-l+1)} \bar{C}_{i}(r-l+1) \\
& \quad \times x\left(r-l+1-\tau_{i}(r-l+1)\right) \\
& \quad-G(r+1) \sum_{l=0}^{M} \Phi_{\sigma(r-l+1)} D(r-l+1) v(r-l+1) \\
& \quad+G(r+1) \sum_{l=0}^{M-1} \sum_{i=1}^{M} \Phi_{\sigma(r-l+1)} \bar{C}_{i}(r-l+1) \\
& \quad \times \hat{x}(r-l+1-d \mid r-l-d) .
\end{aligned}
$$

Before proceeding, we introduce the following lemma.

Lemma 1: [36] Let $F, U, S$ and $T$ be matrices with $S S^{T} \leq$ $I$. For any symmetric matrix $P>0$ and scalar $\alpha>0$, if $\alpha^{-1} I-T P T^{T}>0$ is satisfied, then we have

$$
\begin{aligned}
& (F+U S T) P(F+U S T)^{T} \\
\leq & F\left(P^{-1}-\alpha T^{T} T\right)^{-1} F^{T}+\alpha^{-1} U U^{T} .
\end{aligned}
$$

Lemma 2: The one-step prediction error covariance

$$
P(r+1 \mid r) \triangleq \mathbb{E}\left\{e(r+1 \mid r) e^{T}(r+1 \mid r)\right\}
$$

and the estimator error covariance

$$
P(r+1 \mid r+1) \triangleq \mathbb{E}\left\{e(r+1 \mid r+1) e^{T}(r+1 \mid r+1)\right\}
$$

are, respectively, computed as

$$
\begin{aligned}
P(r+1 \mid r)= & {[\tilde{A}(r)+\tilde{F}(r)] P(r \mid r)[\tilde{A}(r)+\tilde{F}(r)]^{T} } \\
& +B(r) Q(r) B^{T}(r)
\end{aligned}
$$

and

$P(r+1 \mid r+1)$ 


$$
\begin{aligned}
= & \left(I-G(r+1) \sum_{i=1}^{M} \bar{C}_{i}(r+1)\right) P(r+1 \mid r) \\
& \times\left(I-G(r+1) \sum_{i=1}^{M} \bar{C}_{i}(r+1)\right)^{T} \\
& +G(r+1) \sum_{i=1}^{M} \sum_{j=1}^{M} \mathbb{E}\left\{\bar{C}_{i}(r+1) e(r+1 \mid r)\right. \\
& \left.\times e^{T}(r+1 \mid r) \bar{C}_{j}^{T}(r+1)\right\} G^{T}(r+1) \\
& +G(r+1) \sum_{l=0}^{M-1} \sum_{i=1}^{M} \sum_{h=0}^{M-1} \sum_{j=1}^{M} \mathbb{E}\left\{\Phi_{\sigma(r-l+1)}\right. \\
& \times \bar{C}_{i}(r-l+1) x\left(r-l+1-\tau_{i}(r-l+1)\right) \\
& \times x^{T}\left(r-h+1-\tau_{j}(r-h+1)\right) \bar{C}_{j}^{T}(r-h+1) \\
& \left.\times \Phi_{\sigma(r-h+1)}^{T}\right\} G^{T}(r+1)+G(r+1) \\
& \quad M-1 M-1 \\
& \times \sum_{l=0}^{M} \sum_{h=0} \mathbb{E}\left\{\Phi_{\sigma(r-l+1)} D(r-l+1) v(r-l+1)\right. \\
& \left.\times v^{T}(r-h+1) D^{T}(r-h+1) \Phi_{\sigma(r-h+1)}^{T}\right\} G^{T}(r+1) \\
& +G(r+1) \sum_{l=0}^{M-1} \sum_{i=1}^{M} \sum_{h=0}^{M-1} \sum_{j=1}^{M} \Phi_{\sigma(r-l+1)} \\
& \times \bar{C}_{i}(r-l+1) \hat{x}(r-l+1-d \mid r-l-d) \\
& \times \hat{x}^{T}(r-h+1-d \mid r-h-d) \bar{C}_{j}^{T}(r-h+1) \\
& \times \Phi_{\sigma(r-h+1)}^{T} G^{T}(r+1) \\
& +\mathbb{E}\left\{\mathcal{T}_{1} \mathcal{T}_{2}^{T}+\mathcal{T}_{2} \mathcal{T}_{1}^{T}-\mathcal{T}_{1} \mathcal{T}_{3}^{T}-\mathcal{T}_{3} \mathcal{T}_{1}^{T}-\mathcal{T}_{1} \mathcal{T}_{4}^{T}\right. \\
& -\mathcal{T}_{4} \mathcal{T}_{1}^{T}+\mathcal{T}_{1} \mathcal{T}_{5}^{T}+\mathcal{T}_{5} \mathcal{T}_{1}^{T}-\mathcal{T}_{2} \mathcal{T}_{3}^{T}-\mathcal{T}_{3} \mathcal{T}_{2}^{T}+\mathcal{T}_{2} \mathcal{T}_{5}^{T}+\mathcal{T}_{5} \mathcal{T}_{2}^{T}-\mathcal{T}_{3} \mathcal{T}_{5}^{T} \\
& \left.-\mathcal{T}_{5} \mathcal{T}_{3}^{T}-\mathcal{T}_{4} \mathcal{T}_{5}^{T}-\mathcal{T}_{5} \mathcal{T}_{4}^{T}\right\} \\
& \\
&
\end{aligned}
$$

where

$$
\begin{aligned}
\tilde{A}(r) \triangleq & A(r)+(W \otimes \Gamma), \\
\tilde{F}(r) \triangleq & F(r)+U(r) S(r) T(r), \\
\mathcal{T}_{1} \triangleq & \left(I-G(r+1) \sum_{i=1}^{M} \bar{C}_{i}(r+1)\right) e(r+1 \mid r), \\
\mathcal{T}_{2} \triangleq & G(r+1) \sum_{i=1}^{M} \bar{C}_{i}(r+1) e(r+1 \mid r), \\
\mathcal{T}_{3} \triangleq & G(r+1) \sum_{l=0}^{M-1} \sum_{i=1}^{M} \Phi_{\sigma(r-l+1)} \bar{C}_{i}(r-l+1) \\
& \times x\left(r-l+1-\tau_{i}(r-l+1)\right), \\
\mathcal{T}_{4} \triangleq & G(r+1) \sum_{l=0}^{M} \Phi_{\sigma(r-l+1)} D(r-l+1) v(r-l+1), \\
\mathcal{T}_{5} \triangleq & G(r+1) \sum_{l=0}^{M-1} \sum_{i=1}^{M} \Phi_{\sigma(r-l+1)} \bar{C}_{i}(r-l+1) \\
& \times \hat{x}(r-l+1-d \mid r-l-d) .
\end{aligned}
$$

Proof: According to (8) and (10), the equalities (12) and (13) can be readily obtained, and the proof of this lemma is thus omitted.
On the basis of the above preliminaries, we are now ready to present our main results in the following theorem.

Theorem 1: Let $\varepsilon_{i}(i=0,1, \ldots, 10)$ and $\alpha(r)$ be positive scalars. Under the initial condition $\Theta(0 \mid 0)=P(0 \mid 0)>0$, assume that the following two recursive matrix equations

$$
\begin{aligned}
\Theta(r+1 \mid r)= & \left(1+\varepsilon_{0}\right) \tilde{A}(r) \Theta(r \mid r) \tilde{A}(r)^{T}+\left(1+\varepsilon_{0}^{-1}\right) \\
& \times\left(F(r)\left[\Theta^{-1}(r \mid r)-\alpha(r) T^{T}(r) T(r)\right]^{-1}\right. \\
& \left.\times F^{T}(r)+\alpha^{-1}(r) U(r) U^{T}(r)\right) \\
& +B(r) Q(r) B^{T}(r)
\end{aligned}
$$

and

$$
\begin{aligned}
\Theta(r+ & 1 \mid r+1) \\
= & \epsilon_{1}\left(I-G(r+1) \sum_{i=1}^{M} \bar{C}_{i}(r+1)\right) \Theta(r+1 \mid r) \\
& \times\left(I-G(r+1) \sum_{i=1}^{M} \bar{C}_{i}(r+1)\right)^{T} \\
& +\epsilon_{2} M G(r+1) \sum_{i=1}^{M} \bar{C}_{i}(r+1) \Theta(r+1 \mid r) \\
& \times \bar{C}_{i}^{T}(r+1) G^{T}(r+1) \\
& +\epsilon_{3} M^{2} G(r+1) \sum_{l=0}^{M-1} \sum_{i=1}^{M} \sum_{s=0}^{q} p_{s} \Phi_{\sigma(r-l+1)} \\
& \times \bar{C}_{i}(r-l+1) \tilde{X}(r-l+1-s) \bar{C}_{i}^{T}(r-l+1) \\
& \times \Phi_{\sigma(r-l+1)}^{T} G^{T}(r+1) \\
& +\epsilon_{4} M G(r+1) \sum_{l=0}^{M} \Phi_{\sigma(r-l+1)} D(r-l+1) \\
& \times R(r-l+1) D^{T}(r-l+1) \Phi_{\sigma(r-l+1)}^{T} G^{T}(r+1) \\
& +\epsilon_{5} M^{2} G(r+1) \sum_{l=0}^{M-1} \sum_{i=1}^{M} \Phi_{\sigma(r-l+1)} \bar{C}_{i}(r-l+1) \\
& \times \hat{x}(r-l+1-d \mid r-l-d) \\
& \times \hat{x}^{T}(r-l+1-d \mid r-l-d) \bar{C}_{i}^{T}(r-l+1) \\
& \times \Phi_{\sigma(r-l+1)}^{T} G^{T}(r+1),
\end{aligned}
$$

where

$$
\begin{aligned}
\epsilon_{1} \triangleq & 1+\varepsilon_{1}+\varepsilon_{2}+\varepsilon_{3}+\varepsilon_{4}, \\
\epsilon_{2} \triangleq & 1+\varepsilon_{1}^{-1}+\varepsilon_{5}+\varepsilon_{6}+\varepsilon_{7}, \\
\epsilon_{3} \triangleq & 1+\varepsilon_{2}^{-1}+\varepsilon_{5}^{-1}+\varepsilon_{8}, \\
\epsilon_{4} \triangleq & 1+\varepsilon_{3}^{-1}+\varepsilon_{6}^{-1}+\varepsilon_{9}, \\
\epsilon_{5} \triangleq & 1+\varepsilon_{4}^{-1}+\varepsilon_{7}^{-1}+\varepsilon_{8}^{-1}+\varepsilon_{9}^{-1}, \\
\tilde{X}(r-l+1-s) \triangleq & \left(1+\varepsilon_{10}\right) \Theta(r-l+1-s \mid r-l-s) \\
& +\left(1+\varepsilon_{10}^{-1}\right) \hat{x}(r-l+1-s \mid r-l-s) \\
& \times \hat{x}^{T}(r-l+1-s \mid r-l-s),
\end{aligned}
$$

have solutions $\Theta(r+1 \mid r)>0$ and $\Theta(r \mid r)>0$ subject to the constraint

$$
\alpha^{-1}(r) I-T(r) \Theta(r \mid r) T^{T}(r)>0
$$


Then, $\Theta(r+1 \mid r+1)$ is an upper bound of $P(r+1 \mid r+1)$, i.e.,

$$
P(r+1 \mid r+1) \leq \Theta(r+1 \mid r+1)
$$

Proof: This theorem is proved by mathematical induction. First, it follows from the initial condition that $P(0 \mid 0) \leq$ $\Theta(0 \mid 0)$. Assuming $P(r \mid r) \leq \Theta(r \mid r)$, we need to prove $P(r+1 \mid r+1) \leq \Theta(r+1 \mid r+1)$.

To begin with, we rewritten (12) as follows:

$$
\begin{aligned}
P(r+1 \mid r)= & \tilde{A}(r) P(r \mid r) \tilde{A}^{T}(r)+\tilde{F}(r) P(r \mid r) \tilde{F}^{T}(r) \\
& +\tilde{A}(r) P(r \mid r) \tilde{F}^{T}(r)+\tilde{F}(r) P(r \mid r) \tilde{A}^{T}(r) \\
& +B(r) Q(r) B^{T}(r) .
\end{aligned}
$$

Applying Lemma 1 to the second term on the right-hand side of above equality, one has

$$
\begin{aligned}
\tilde{F}(r) P(r \mid r) \tilde{F}^{T}(r) \leq & F(r)\left[P^{-1}(r \mid r)-\alpha(r) T^{T}(r) T(r)\right]^{-1} \\
& \times F^{T}(r)+\alpha^{-1}(r) U(r) U^{T}(r) .
\end{aligned}
$$

Utilizing the elementary inequality $a b^{T}+b a^{T} \leq \varepsilon a a^{T}+$ $\varepsilon^{-1} b b^{T}$ (where $a, b$ are arbitrary vectors, and $\varepsilon$ is an arbitrary positive scalar) and the assumption of $P(r \mid r) \leq \Theta(r \mid r)$, we derive that

$$
\begin{aligned}
P(r+1 \mid r) \leq & \left(1+\varepsilon_{0}\right) \tilde{A}(r) \Theta(r \mid r) \tilde{A}(r)^{T}+\left(1+\varepsilon_{0}^{-1}\right) \\
& \times\left(F(r)\left[\Theta^{-1}(r \mid r)-\alpha(r) T^{T}(r) T(r)\right]^{-1}\right. \\
& \left.\times F^{T}(r)+\alpha^{-1}(r) U(r) U^{T}(r)\right) \\
& +B(r) Q(r) B^{T}(r)
\end{aligned}
$$

Next, applying the elementary inequality to the last term of the right-hand side of (13), we have

$$
\begin{aligned}
\mathbb{E}\left\{\mathcal{T}_{2} \mathcal{T}_{2}^{T}\right\}= & G(r+1) \sum_{i=1}^{M} \sum_{j=1}^{M} \mathbb{E}\left\{\bar{C}_{i}(r+1) e(r+1 \mid r)\right. \\
& \left.\times e^{T}(r+1 \mid r) \bar{C}_{j}^{T}(r+1)\right\} G^{T}(r+1) \\
\leq & M G(r+1) \sum_{i=1}^{M} \bar{C}_{i}(r+1) P(r+1 \mid r) \\
& \times \bar{C}_{i}^{T}(r+1) G^{T}(r+1), \\
\mathbb{E}\left\{\mathcal{T}_{3} \mathcal{T}_{3}^{T}\right\}= & G(r+1) \sum_{l=0}^{M-1} \sum_{i=1}^{M} \sum_{h=0}^{M-1} \sum_{j=1}^{M} \mathbb{E}\left\{\Phi_{\sigma(r-l+1)}\right. \\
& \times \bar{C}_{i}(r-l+1) x\left(r-l+1-\tau_{i}(r-l+1)\right) \\
& \times x^{T}\left(r-h+1-\tau_{j}(r-h+1)\right) \\
& \left.\times \bar{C}_{j}^{T}(r-h+1) \Phi_{\sigma(r-h+1)}^{T}\right\} G^{T}(r+1) \\
\leq & M^{2} G(r+1) \sum_{l=0}^{M-1} \sum_{i=1}^{M} \sum_{s=0}^{q} p_{s} \Phi_{\sigma(r-l+1)} \\
& \times \bar{C}_{i}(r-l+1) \mathbb{E}\left\{x\left(r-l+1-\tau_{i}(r-l+1)\right)\right. \\
& \left.\times x^{T}\left(r-l+1-\tau_{i}(r-l+1)\right)\right\} \\
& \times \bar{C}_{i}^{T}(r-l+1) \Phi_{\sigma(r-l+1)}^{T} G^{T}(r+1),
\end{aligned}
$$

$$
\begin{aligned}
\mathbb{E}\left\{\mathcal{T}_{4} \mathcal{T}_{4}^{T}\right\}= & G(r+1) \sum_{l=0}^{M-1} \sum_{h=0}^{M-1} \mathbb{E}\left\{\Phi_{\sigma(r-l+1)} D(r-l+1)\right. \\
& \times v(r-l+1) v^{T}(r-h+1) D^{T}(r-h+1) \\
& \left.\times \Phi_{\sigma(r-h+1)}^{T}\right\} G^{T}(r+1) \\
\leq & M G(r+1) \sum_{l=0}^{M-1} \Phi_{\sigma(r-l+1)} D(r-l+1) \\
& \times R(r-l+1) D^{T}(r-l+1) \\
& \times \Phi_{\sigma(r-l+1)}^{T} G^{T}(r+1) \\
\mathbb{E}\left\{\mathcal{T}_{5} \mathcal{T}_{5}^{T}\right\}= & G(r+1) \sum_{l=0}^{M-1} \sum_{i=1}^{M} \sum_{h=0}^{M-1} \sum_{j=1}^{M} \Phi_{\sigma(r-l+1)} \\
& \times \bar{C}_{i}(r-l+1) \hat{x}(r-l+1-d \mid r-l-d) \\
& \times \hat{x}^{T}(r-h+1-d \mid r-h-d) \\
& \times \bar{C}_{j}^{T}(r-h+1) \Phi_{\sigma(r-h+1)}^{T} G^{T}(r+1) \\
\leq & M^{2} G(r+1) \sum_{l=0}^{M-1} \sum_{i=1}^{M} \Phi_{\sigma(r-l+1)} \\
& \times \bar{C}_{i}(r-l+1) \hat{x}(r-l+1-d \mid r-l-d) \\
& \times \hat{x}^{T}(r-l+1-d \mid r-l-d) \\
& \times \bar{C}_{i}^{T}(r-l+1) \Phi_{\sigma(r-l+1)}^{T} G^{T}(r+1) .
\end{aligned}
$$

Combining $x(r)=e(r+1 \mid r)+\hat{x}(r+1 \mid r)$ and the elementary inequality yields

$$
\begin{aligned}
& \mathbb{E}\left\{x\left(r-l+1-\tau_{i}(r-l+1)\right) x^{T}\left(r-l+1-\tau_{i}(r-l+1)\right)\right\} \\
\leq & \left(1+\varepsilon_{10}\right) \sum_{s=0}^{q} p_{s} P(r-l+1-s \mid r-l-s) \\
& +\left(1+\varepsilon_{10}^{-1}\right) \sum_{s=0}^{q} p_{s} \hat{x}(r-l+1-s \mid r-l-s) \\
& \times \hat{x}^{T}(r-l+1-s \mid r-l-s) .
\end{aligned}
$$

Consequently, (18) can be handled by

$$
\begin{aligned}
& \mathbb{E}\left\{\mathcal{T}_{3} \mathcal{T}_{3}^{T}\right\} \\
\leq & M^{2} G(r+1) \sum_{l=0}^{M-1} \sum_{i=1}^{M} \sum_{s=0}^{q} p_{s} \Phi_{\sigma(r-l+1)} \bar{C}_{i}(r-l+1) \\
& \times\left(\left(1+\varepsilon_{10}\right) \sum_{s=0}^{q} p_{s} P(r-l+1-s \mid r-l-s)\right. \\
& +\left(1+\varepsilon_{10}^{-1}\right) \sum_{s=0}^{q} p_{s} \hat{x}(r-l+1-s \mid r-l-s) \\
& \left.\times \hat{x}^{T}(r-l+1-s \mid r-l-s)\right) \\
& \times \bar{C}_{i}^{T}(r-l+1) \Phi_{\sigma(r-l+1)}^{T} G^{T}(r+1) .
\end{aligned}
$$

From (16)-(20), one has

$$
\begin{aligned}
& P(r+1 \mid r+1) \\
\leq & \epsilon_{1}\left(I-G(r+1) \sum_{i=1}^{M} \bar{C}_{i}(r+1)\right) P(r+1 \mid r) \\
& \times\left(I-G(r+1) \sum_{i=1}^{M} \bar{C}_{i}(r+1)\right)^{T}
\end{aligned}
$$




$$
\begin{aligned}
& +\epsilon_{2} M G(r+1) \sum_{i=1}^{M} \bar{C}_{i}(r+1) P(r+1 \mid r) \\
& \times \bar{C}_{i}^{T}(r+1) G^{T}(r+1) \\
& +\epsilon_{3} M^{2} G(r+1) \sum_{l=0}^{M-1} \sum_{i=1}^{M} \sum_{s=0}^{q} p_{s} \Phi_{\sigma(r-l+1)} \\
& \times \bar{C}_{i}(r-l+1) \tilde{X}(r-l+1-s) \\
& \times \bar{C}_{i}^{T}(r-l+1) \Phi_{\sigma(r-l+1)}^{T} G^{T}(r+1) \\
& +\epsilon_{4} M G(r+1) \sum_{l=0}^{M-1} \Phi_{\sigma(r-l+1)} D(r-l+1) \\
& \times R(r-l+1) D^{T}(r-l+1) \Phi_{\sigma(r-l+1)}^{T} G^{T}(r+1) \\
& +\epsilon_{5} M^{2} G(r+1) \sum_{l=0}^{M-1} \sum_{i=1}^{M} \Phi_{\sigma(r-l+1)} \\
& \times \bar{C}_{i}(r-l+1) \hat{x}(r-l+1-d \mid r-l-d) \\
& \times \hat{x}^{T}(r-l+1-d \mid r-l-d) \\
& \times \bar{C}_{i}^{T}(r-l+1) \Phi_{\sigma(r-l+1)}^{T} G^{T}(r+1) .
\end{aligned}
$$

According to (14)-(16) and (21), we conclude

$$
P(r+1 \mid r+1) \leq \Theta(r+1 \mid r+1)
$$

which ends the proof.

In Theorem 1, an upper bound of estimation error covariance is presented. Now, we are going to determine the estimator gain by locally minimize the obtained upper bound.

Theorem 2: Design the estimator parameter as follows:

$$
G(r+1)=\epsilon_{1} \Theta(r+1 \mid r) \sum_{i=1}^{M} \bar{C}_{i}^{T}(r+1) \Psi^{-1}(r+1),
$$

where

$$
\begin{aligned}
& \Psi(r+1) \\
\triangleq & \epsilon_{1}\left(\sum_{i=1}^{M} \bar{C}_{i}(r+1)\right) \Theta(r+1 \mid r)\left(\sum_{i=1}^{M} \bar{C}_{i}(r+1)\right)^{T} \\
& +\epsilon_{2} M \sum_{i=1}^{M} \bar{C}_{i}(r+1) \Theta(r+1 \mid r) \bar{C}_{i}^{T}(r+1) \\
& +\epsilon_{3} M^{2} \sum_{l=0}^{M-1} \sum_{i=1}^{M} \sum_{s=0}^{q} p_{s} \Phi_{\sigma(r-l+1)} \bar{C}_{i}(r-l+1) \\
& \times \tilde{X}(r-l+1-s) \bar{C}_{i}^{T}(r-l+1) \Phi_{\sigma(r-l+1)}^{T} \\
& +\epsilon_{4} M \sum_{l=0}^{M-1} \Phi_{\sigma(r-l+1)} D(r-l+1) \\
& \times R(r-l+1) D^{T}(r-l+1) \Phi_{\sigma(r-l+1)}^{T} \\
& +\epsilon_{5} M^{2} \sum_{l=0}^{M-1} \sum_{i=1}^{M} \Phi_{\sigma(r-l+1)} \bar{C}_{i}(r-l+1) \\
& \times \hat{x}(r-l+1-d \mid r-l-d) \\
& \times \hat{x}^{T}(r-l+1-d \mid r-l-d) \\
& \times \bar{C}_{i}^{T}(r-l+1) \Phi_{\sigma(r-l+1)}^{T} .
\end{aligned}
$$

Then, the upper bound matrix $P(r+1 \mid r+1)$ is minimized by

$$
\Theta(r+1 \mid r+1)
$$

$$
\begin{aligned}
& =\epsilon_{1} \Theta(r+1 \mid r)-\epsilon_{1}^{2} \Theta(r+1 \mid r)\left(\sum_{i=1}^{M} \bar{C}_{i}(r+1)\right)^{T} \\
& \times \Psi^{-1}(r+1)\left(\sum_{i=1}^{M} \bar{C}_{i}(r+1)\right) \Theta(r+1 \mid r) .
\end{aligned}
$$

Proof: The upper bound derived in Theorem 1 can be rewritten as the following form:

$$
\begin{aligned}
& \Theta(r+1 \mid r+1) \\
= & {\left[G(r+1)-\epsilon_{1} \Theta(r+1 \mid r) \sum_{i=1}^{M} \bar{C}_{i}^{T}(r+1) \Psi^{-1}(r+1)\right] } \\
& \times \Psi(r+1)\left[G(r+1)-\epsilon_{1} \Theta(r+1 \mid r) \sum_{i=1}^{M} \bar{C}_{i}^{T}(r+1)\right. \\
& \left.\times \Psi^{-1}(r+1)\right]^{T}+\epsilon_{1} \Theta(r+1 \mid r) \\
& -\epsilon_{1}^{2} \Theta(r+1 \mid r)\left(\sum_{i=1}^{M} \bar{C}_{i}(r+1)\right)^{T} \Psi^{-1}(r+1) \\
& \times\left(\sum_{i=1}^{M} \bar{C}_{i}(r+1)\right) \Theta(r+1 \mid r) .
\end{aligned}
$$

It is easy to see that the minimization of the upper bound (15) can be obtained when the estimator parameter is selected as (22), and the proof is complete.

\section{Boundedness AnAlysis}

To further analyze the boundedness of the estimation error, a definition concerning the boundedness of a stochastic process and some reasonable assumptions are introduced.

Definition 1: [28] The stochastic process $\zeta(r)$ is said to be exponentially mean-square bounded, if there exist real numbers $\alpha>0, \beta>0$ and $0<\kappa<1$ such that

$$
\mathbb{E}\{\|\zeta(r)\|\} \leq \alpha\|\zeta(0)\|^{2} \kappa^{r}+\beta
$$

holds for all $r \geq 0$.

Assumption 1: There exist some positive real scalars $\bar{a}, \underline{b}, \bar{b}, \underline{c}, \bar{c}, \bar{d}, \bar{f}, \bar{u}, \bar{t}, \bar{\gamma}, \bar{\iota}, \bar{\chi}, \underline{\theta}, \bar{\theta}, \underline{q}, \bar{q}$ and $\bar{\pi}$ such that the following conditions

$$
\begin{aligned}
& \|A(r)\| \leq \bar{a}, \quad \underline{b} \leq\|B(r)\| \leq \bar{b}, \quad\|D(r)\| \leq \bar{d} \\
& \|F(r)\| \leq \bar{f}, \quad\|U(r)\| \leq \bar{u}, \quad\|T(r)\| \leq \bar{t}, \\
& \|W \otimes \Gamma\| \leq \bar{\gamma}, \quad\|\hat{x}(r+1 \mid r)\| \leq \bar{\iota}, \quad \tilde{X}(r) \leq \bar{\chi} I \\
& \underline{c}^{2} I \leq \bar{C}_{i}(r) \bar{C}_{i}^{T}(r) \leq \bar{c}^{2} I, \quad R(r) \leq \bar{\pi} I \\
& \underline{\theta} I \leq \Theta(r+1 \mid r) \leq \bar{\theta} I, \quad \underline{q} I \leq Q(r) \leq \bar{q} I \\
& (\bar{a}+\bar{\gamma})^{2}<1
\end{aligned}
$$

hold for $i=1,2, \ldots, M$.

For simplicity, some notations are presented as follows:

$$
\begin{aligned}
& \bar{g} \triangleq \frac{\bar{\theta} \bar{c}}{\underline{\theta} \underline{c}^{2}}, \\
& \varphi \triangleq \bar{b}^{2} \bar{q}+\varrho, \\
& \phi \triangleq \bar{a}+\bar{\gamma}+\bar{f}+\bar{u} \bar{t}, \\
& \bar{\chi} \triangleq\left(1+\varepsilon_{10}\right) \bar{\theta}+\left(1+\varepsilon_{10}^{-1}\right) \bar{\iota}^{2},
\end{aligned}
$$




$$
\begin{aligned}
\bar{z} \triangleq & \left(1+\xi_{1}\right) \bar{\chi} \bar{c}^{2} \bar{g}^{2} m M^{5}+\left(1+\xi_{2}\right) v \bar{\pi} \bar{d}^{2} \bar{g}^{2} M^{2} \\
& +\left(1+\xi_{1}^{-1}+\xi_{2}^{-1}\right) \bar{\iota}^{2} \bar{c}^{2} \bar{g}^{2} M^{4} \\
\mu \triangleq & (1+\eta)\left[1-\left(1+\frac{\phi^{2} \bar{\omega}}{\underline{b}^{2} \underline{q}}\right)^{-1}\right] \\
\nu \triangleq & \frac{w \bar{q} \bar{b}^{2}}{\varrho}+\left(1+\eta^{-1}\right) \frac{\bar{z}}{\varrho}
\end{aligned}
$$

where $\varrho, \xi_{1}, \xi_{2}, \eta, \bar{\omega}$ are positive scalars.

Theorem 3: Consider the discrete-time complex network described in (2a)-(2b) with the designed estimator (5a)-(5b). Then, under Assumption 1, the estimation error is exponentially mean-square bounded.

Proof: Substituting (8) to (9) yields

$$
e(r+1 \mid r+1)=[\tilde{A}(r)+\tilde{F}(r)] e(r \mid r)+\tilde{w}(r)+\tilde{z}(r),
$$

where

$$
\begin{aligned}
\tilde{w}(r) \triangleq & B(r) w(r), \\
\tilde{z}(r) \triangleq & -G(r+1) \sum_{l=0}^{M-1} \sum_{i=1}^{M} \Phi_{\sigma(r-l+1)} \bar{C}_{i}(r-l+1) \\
& \times x\left(r-l+1-\tau_{i}(r-l+1)\right) \\
& -G(r+1) \sum_{l=0}^{M} \Phi_{\sigma(r-l+1)} D(r-l+1) v(r-l+1) \\
& +G(r+1) \sum_{l=0}^{M-1} \sum_{i=1}^{M} \Phi_{\sigma(r-l+1)} \bar{C}_{i}(r-l+1) \\
& \times \hat{x}(r-l+1-d \mid r-l-d) .
\end{aligned}
$$

From (21), it follows immediately that

$$
\begin{aligned}
& \|G(r+1)\| \\
= & \left\|\epsilon_{1} \Theta(r+1 \mid r) \sum_{i=1}^{M} \bar{C}_{i}^{T}(r+1) \Psi^{-1}(r+1)\right\| \\
\leq & \| \epsilon_{1} \Theta(r+1 \mid r) \sum_{i=1}^{M} \bar{C}_{i}^{T}(r+1)\left[\epsilon_{1}\left(\sum_{i=1}^{M} \bar{C}_{i}(r+1)\right)\right. \\
& \left.\times \Theta(r+1 \mid r)\left(\sum_{i=1}^{M} \bar{C}_{i}(r+1)\right)^{T}\right]^{-1} \| \\
\leq & \frac{\bar{\theta} \bar{c}}{\underline{\theta} \underline{c}^{2}}=\bar{g} .
\end{aligned}
$$

By resorting to Assumption 1 and the properties of the trace, one has

$$
\begin{aligned}
\mathbb{E}\left\{\tilde{w}^{T}(r) \tilde{w}(r)\right\} & =\mathbb{E}\left\{w^{T}(r) B^{T}(r) B(r) w(r)\right\} \\
& =\operatorname{tr}\left\{\mathbb{E}\left\{w(r) w^{T}(r)\right\} B^{T}(r) B(r)\right\} \\
& \leq w \bar{q} \bar{b}^{2}
\end{aligned}
$$

and

$$
\begin{aligned}
& \mathbb{E}\left\{\tilde{z}^{T}(r) \tilde{z}(r)\right\} \\
\leq & \left(1+\xi_{1}\right) \sum_{l=0}^{M-1} \sum_{i=1}^{M} \sum_{h=0}^{M-1} \sum_{j=1}^{M} \mathbb{E}\left\{x^{T}\left(r-l+1-\tau_{i}(r-l+1)\right)\right. \\
& \times \bar{C}_{i}^{T}(r-l+1) \Phi_{\sigma(r-l+1)}^{T} G^{T}(r+1) G(r+1)
\end{aligned}
$$

$$
\begin{aligned}
& \left.\times \Phi_{\sigma(r-h+1)} \bar{C}_{j}(r-h+1) x\left(r-h+1-\tau_{j}(r-h+1)\right)\right\} \\
& +\left(1+\xi_{2}\right) \sum_{l=0}^{M-1} \sum_{h=0}^{M-1} \mathbb{E}\left\{v^{T}(r-l+1) D^{T}(r-l+1)\right. \\
& \times \Phi_{\sigma(r-l+1)}^{T} G^{T}(r+1) G(r+1) \Phi_{\sigma(r-h+1)} \\
& \times D(r-h+1) v(r-h+1)\} \\
& +\left(1+\xi_{1}^{-1}+\xi_{2}^{-1}\right) \\
& \times \sum_{l=0}^{M-1} \sum_{i=1}^{M} \sum_{h=0}^{M-1} \sum_{j=1}^{M} \hat{x}^{T}(r-l+1-d \mid r-l-d) \\
& \times \bar{C}_{i}^{T}(r-l+1) \Phi_{\sigma(r-l+1)}^{T} G^{T}(r+1) G(r+1) \\
& \times \Phi_{\sigma(r-h+1)} \bar{C}_{j}(r-h+1) \hat{x}^{T}(r-h+1-d \mid r-h-d) \\
& \leq\left(1+\xi_{1}\right) M^{2} \sum_{l=0}^{M-1} \sum_{i=1}^{M} \sum_{s=0}^{q} p_{s} \operatorname{tr}\{\tilde{X}(r-l+1-s) \\
& \times \bar{C}_{i}^{T}(r-l+1) \Phi_{\sigma(r-l+1)}^{T} G^{T}(r+1) G(r+1) \\
& \left.\times \Phi_{\sigma(r-l+1)} \bar{C}_{i}(r-l+1)\right\}+\left(1+\xi_{2}\right) M \\
& \times \sum_{l=0}^{M-1} \operatorname{tr}\left\{R(r-l+1) D^{T}(r-l+1) \Phi_{\sigma(r-l+1)}^{T}\right. \\
& \left.\times G^{T}(r+1) G(r+1) \Phi_{\sigma(r-l+1)} D(r-l+1)\right\} \\
& +\left(1+\xi_{1}^{-1}+\xi_{2}^{-1}\right) M^{2} \\
& \times \sum_{l=0}^{M-1} \sum_{i=1}^{M} \operatorname{tr}\left\{\hat{x}^{T}(r-l+1-d \mid r-l-d)\right. \\
& \times \bar{C}_{i}^{T}(r-l+1) \Phi_{\sigma(r-l+1)}^{T} G^{T}(r+1) G(r+1) \\
& \left.\times \Phi_{\sigma(r-l+1)} \bar{C}_{i}(r-l+1) \hat{x}(r-l+1-d \mid r-l-d)\right\} \\
& \leq\left(1+\xi_{1}\right) \bar{\chi} \bar{c}^{2} \bar{g}^{2} m M^{5}+\left(1+\xi_{2}\right) v \bar{\pi} \bar{d}^{2} \bar{g}^{2} M^{2} \\
& +\left(1+\xi_{1}^{-1}+\xi_{2}^{-1}\right) \bar{\iota}^{2} \bar{c}^{2} \bar{g}^{2} M^{4} \\
& =\bar{z} \text {. }
\end{aligned}
$$

Now, let us consider the following iterative matrix equation with respect to $\Omega(r)$ :

$$
\begin{aligned}
\Omega(r+1)= & {[\tilde{A}(r)+\tilde{F}(r)] \Omega(r) \tilde{A}^{T}(r)+B(r) Q(r) B^{T}(r) } \\
& +\varrho I,
\end{aligned}
$$

where the initial value is $\Omega(0)=B(0) Q(0) B^{T}(0)+\varrho I$.

It follows from (27) that

$$
\begin{aligned}
& \|\Omega(r+1)\| \\
\leq & \|\tilde{A}(r)+\tilde{F}(r)\|^{2}\|\Omega(r)\|+\|B(r)\|^{2}\|Q(r)\|+\|\varrho I\| \\
\leq & (\|A(r)\|+\|W \otimes \Gamma\|+\|F(r)\|+\|U(r) S(r) T(r)\|)^{2} \\
& \times\|\Omega(r)\|+\|B(r)\|^{2}\|Q(r)\|+\|\varrho I\| \\
\leq & (\bar{a}+\bar{\gamma}+\bar{f}+\bar{u} \bar{t})^{2}\|\Omega(r)\|+\bar{b}^{2} \bar{q}+\varrho \\
= & \phi^{2}\|\Omega(r)\|+\varphi
\end{aligned}
$$

and therefore

$$
\|\Omega(r)\| \leq \phi^{2 r}\|\Omega(0)\|+\varphi \sum_{i=0}^{r-1} \phi^{2 i} .
$$

It can be seen from $\phi^{2}<1$ that

$$
\|\Omega(r)\| \leq\|\Omega(0)\|+\varphi \sum_{i=0}^{\infty} \phi^{2 i}
$$




$$
=\|\Omega(0)\|+\frac{\varphi}{1-\phi^{2}} .
$$

On the other hand, it is easy to see that

$$
\Omega(r) \geq \varrho I .
$$

According to (28)-(29), there exists a scalar $\bar{\omega}>0$ such that $\varrho I \leq \Omega(r) \leq \bar{\omega} I$.

Let $V_{r}(e(r \mid r)) \triangleq e^{T}(r \mid r) \Omega^{-1}(r) e(r \mid r)$. From (23), it can be deduced that

$$
\begin{aligned}
& \mathbb{E}\left\{V_{r+1}(e(r+1 \mid r+1)) \mid e(r \mid r)\right\}-(1+\eta) V_{r}(e(r \mid r)) \\
= & \mathbb{E}\left\{[(\tilde{A}(r)+\tilde{F}(r)) e(r \mid r)+\tilde{w}(r)+\tilde{z}(r)]^{T} \Omega^{-1}(r+1)\right. \\
& \times[(\tilde{A}(r)+\tilde{F}(r)) e(r \mid r)+\tilde{w}(r)+\tilde{z}(r)]\} \\
& -(1+\eta) e^{T}(r \mid r) \Omega^{-1}(r) e(r \mid r) \\
\leq & (1+\eta) \mathbb{E}\left\{e ^ { T } ( r | r ) \left[(\tilde{A}(r)+\tilde{F}(r))^{T} \Omega^{-1}(r+1)\right.\right. \\
& \left.\left.\times(\tilde{A}(r)+\tilde{F}(r))-\Omega^{-1}(r)\right] e(r \mid r)\right\} \\
& +\left(1+\eta^{-1}\right) \mathbb{E}\left\{\tilde{z}^{T}(r) \Omega^{-1}(r+1) \tilde{z}(r)\right\} \\
& +\mathbb{E}\left\{\tilde{w}^{T}(r) \Omega^{-1}(r+1) \tilde{w}(r)\right\} .
\end{aligned}
$$

From the definition of $\Omega(r+1)$ and utilizing the matrix inversion lemma, we obtain that

$$
\begin{aligned}
& (\tilde{A}(r)+\tilde{F}(r))^{T} \Omega^{-1}(r+1)(\tilde{A}(r)+\tilde{F}(r))-\Omega^{-1}(r) \\
= & (\tilde{A}(r)+\tilde{F}(r))^{T}\left[(\tilde{A}(r)+\tilde{F}(r)) \Omega(r)(\tilde{A}(r)+\tilde{F}(r))^{T}\right. \\
& \left.+B(r) Q(r) B^{T}(r)+\varrho I\right]^{-1}(\tilde{A}(r)+\tilde{F}(r))-\Omega^{-1}(r) \\
= & -\left[\Omega(r)+\Omega(r)(\tilde{A}(r)+\tilde{F}(r))^{T}\left(B(r) Q(r) B^{T}(r)\right.\right. \\
& \left.+\varrho I)^{-1}(\tilde{A}(r)+\tilde{F}(r)) \Omega(r)\right]^{-1} \\
= & -\left[I+(\tilde{A}(r)+\tilde{F}(r))^{T}\left(B(r) Q(r) B^{T}(r)+\varrho I\right)^{-1}\right. \\
& \times(\tilde{A}(r)+\tilde{F}(r)) \Omega(r)]^{-1} \Omega^{-1}(r) \\
\leq & -\left(1+\frac{\phi^{2} \bar{\omega}}{\underline{b}^{2} \underline{q}}\right)^{-1} \Omega^{-1}(r) .
\end{aligned}
$$

Substituting (31) into (30) leads to

$$
\begin{aligned}
& \mathbb{E}\left\{V_{r+1}(e(r+1 \mid r+1)) \mid e(r \mid r)\right\}-(1+\eta) V_{r}(e(r \mid r)) \\
\leq & -(1+\eta)\left(1+\frac{\phi^{2} \bar{\omega}}{\underline{b}^{2} \underline{q}}\right)^{-1} V_{r}(e(r \mid r)) \\
& +\frac{w \bar{q} \bar{b}^{2}}{\varrho}+\left(1+\eta^{-1}\right) \frac{\bar{z}}{\varrho} \\
= & \mu V_{r}(e(r \mid r))+\nu
\end{aligned}
$$

and it is easy to verify that $\mu \in(0,1)$ for some $\eta>0$.

Based on the above derivation, we have

$$
\begin{aligned}
& \mathbb{E}\left\{\|e(r+1 \mid r+1)\|^{2}\right\} \\
\leq & \frac{\bar{\omega}}{\varrho} \mathbb{E}\left\{\|e(0 \mid 0)\|^{2}\right\} \mu^{r+1}+\nu \bar{\omega} \sum_{i=0}^{r} \mu^{i} \\
\leq & \frac{\bar{\omega}}{\varrho} \mathbb{E}\left\{\|e(0 \mid 0)\|^{2}\right\} \mu^{r+1}+\nu \bar{\omega} \sum_{i=0}^{\infty} \mu^{i} \\
= & \frac{\bar{\omega}}{\varrho} \mathbb{E}\left\{\|e(0 \mid 0)\|^{2}\right\} \mu^{r+1}+\frac{\nu \bar{\omega}}{1-\mu},
\end{aligned}
$$

and our proof is complete according the definition 1.

Remark 4: In this paper, a recursive SE problem is addressed for a class of SCNs subject to packet disorders. The RR protocol is introduced to alleviate the communication burden. Based on the measurement model suffering from both random delays and RR protocols, a recursive estimator is devised under which an upper bound is first obtained on the estimation error covariance in Theorem 1 and then minimized in Theorem 2. In addition, a sufficient condition is given in Theorem 3 to ensure the exponential mean-square boundedness of the estimation error.

Remark 5: Compared to the existing literature concerning $\mathrm{SE}$ issue, the main results acquired in this paper exhibit the following distinctive features: 1) a comprehensive measurement model is put forward by taking into account the random delays and RR protocols; 2) a sequence of random variables is used to characterize the time delays occurring in the sensing process; 3 ) the influences of the coupling structure between nodes, the packet disorders and the RR protocols on the estimator performance are thoroughly investigated in a quantitative way; and 4) matrix analysis techniques are employed to derive the sufficient condition for mean-square boundedness of estimation error.

\section{NUMERICAL EXAMPLE}

In this section, the validity of developed estimator for the system (2a)-(2b) subject to RR protocols and packet disorders is verified by a numerical example.

Consider a stochastic discrete-time four-node network (1a)(1b) with parameters:

$$
\begin{aligned}
& A_{1}(r)=\left[\begin{array}{cc}
0.31 & -0.31 \cos (r) \\
0.58 & 0.19
\end{array}\right] \text {, } \\
& A_{2}(r)=\left[\begin{array}{cc}
0.39 & -0.39 \sin (r) \\
0.55 & 0.16
\end{array}\right] \text {, } \\
& A_{3}(r)=\left[\begin{array}{cc}
0.38 & -0.51 \sin (r) \\
0.55 & 0.15
\end{array}\right] \text {, } \\
& A_{4}(r)=\left[\begin{array}{cc}
0.39 & -0.71 \cos (r) \\
0.56 & 0.15
\end{array}\right] \text {, } \\
& B_{1}(r)=\left[\begin{array}{c}
0.91 \\
-0.21+0.1 \sin (r)
\end{array}\right], \quad B_{2}(r)=\left[\begin{array}{c}
0.91 \cos (r) \\
-0.12
\end{array}\right], \\
& B_{3}(r)=\left[\begin{array}{c}
0.92 \\
-0.21+0.1 \cos (r)
\end{array}\right], \quad B_{4}(r)=\left[\begin{array}{c}
0.91 \sin (r) \\
-0.2
\end{array}\right], \\
& C_{1}(r)=\left[\begin{array}{ll}
0.3 & -0.15+0.1 \sin (r)
\end{array}\right], \\
& C_{2}(r)=\left[\begin{array}{ll}
0.1+0.1 \cos (r) & 0.6
\end{array}\right], \\
& C_{3}(r)=\left[\begin{array}{ll}
-0.2 & 0.6+0.1 \cos (r)
\end{array}\right] \text {, } \\
& C_{4}(r)=\left[\begin{array}{ll}
-0.2+0.1 \sin (r) & 0.5
\end{array}\right] \text {, } \\
& D_{1}(r)=0.3, \quad D_{2}(r)=0.2, \quad D_{3}(r)=0.4, \quad D_{4}(r)=0.1 .
\end{aligned}
$$

The nonlinear function $f\left(x_{i}(r)\right)$ is described by

$$
f\left(x_{i}(r)\right)=\left[\begin{array}{c}
0.1 \sin \left(x_{i}^{1}(r) x_{i}^{2}(r)\right) \\
0.385 \cos \left(x_{i}^{1}(r) x_{i}^{2}(r)\right)
\end{array}\right]
$$


where $x_{i}(r)=\left[\begin{array}{ll}x_{i}^{1}(r) & x_{i}^{2}(r)\end{array}\right]^{T}(i=1,2,3,4)$ is the system state of the $i$ th node. The coupling configuration matrix is assumed to be $W=\left[\omega_{i j}\right]_{4 \times 4}(i, j=1,2,3,4)$ with

$$
\omega_{i j}=\left\{\begin{array}{rr}
-0.3, & i=j, \\
0.1, & i \neq j
\end{array}\right.
$$

and the inner-coupling matrix is given by $\Gamma=0.2 I_{2}$. The covariances of zero-mean white noises are $Q(r)=0.005$ and $R(r)=0.005$.

In this simulation, the upper bound of the random time delay $\tau_{i}(r)$ is set as $q=3$. The initial mean and covariance of the estimate are given as

$$
\begin{aligned}
& \bar{x}_{1}(0)=\left[\begin{array}{ll}
-0.34 & 0.45
\end{array}\right]^{T}, \quad \bar{x}_{2}(0)=\left[\begin{array}{ll}
-0.24 & 0.55
\end{array}\right]^{T}, \\
& \bar{x}_{3}(0)=\left[\begin{array}{ll}
-0.44 & 0.45
\end{array}\right]^{T}, \quad \bar{x}_{4}(0)=\left[\begin{array}{ll}
-0.34 & 0.35
\end{array}\right]^{T}, \\
& P_{1}(0)=P_{2}(0)=P_{3}(0)=P_{4}(0)=2 I_{2} .
\end{aligned}
$$

The other parameters are chosen as $\alpha=0.5, \varepsilon_{0}=0.5, \varepsilon_{1}=$ $0.25, \varepsilon_{2}=0.05, \varepsilon_{3}=0.25, \varepsilon_{4}=0.05, \varepsilon_{5}=\varepsilon_{6}=\varepsilon_{7}=\varepsilon_{8}=$ $\varepsilon_{9}=\varepsilon_{10}=5$, and the initial conditions are given by

$$
\begin{aligned}
& X(-2)=X(-1)=X(0) \\
& =\left(1+\varepsilon_{10}\right) P(0)+\left(1+\varepsilon_{10}^{-1}\right) \bar{x}(0) \bar{x}^{T}(0), \\
& \hat{x}(-2 \mid-3)=\hat{x}(-1 \mid-2)=\hat{x}(0 \mid-1)=\bar{x}(0) \text {, } \\
& x(-2)=x(-1)=\left[\begin{array}{llllllll}
0 & 0 & 0 & 0 & 0 & 0 & 0 & 0
\end{array}\right]^{T}, \\
& y(0)=\left[\begin{array}{llll}
-0.35 & 0.34 & 0.59 & -0.27
\end{array}\right]^{T} .
\end{aligned}
$$

For $i=1,2,3,4$, the root mean square error (RMSE) of each node is defined by $\operatorname{RMSE}_{i}(r)=$ $\sqrt{\frac{1}{N} \sum_{j=1}^{N}\left(x_{i}^{1, j}(r)-\hat{x}_{i}^{1, j}(r \mid r)\right)^{2}+\left(x_{i}^{2, j}(r)-\hat{x}_{i}^{2, j}(r \mid r)\right)^{2}}$ with $N$ being the times of independent experiments. In this simulation, we set $N=100$.

In terms of the above parameters and Theorems 1-2, an upper bound of the estimation error covariance and the estimator gain can be derived recursively. Simulation result$\mathrm{s}$ are shown in Figs. 2-9. Specifically, Figs. 2-5 plot the trajectories of actual states $x_{i}^{j}(r)$ and their estimates $\hat{x}_{i}^{j}(r)$ $(i=1,2,3,4 ; j=1,2)$, which illustrate that the estimator can estimate the actual state well. In other words, the designed estimator has a satisfactory tracking performance under the RR protocols and packet disorders. To quantify the accuracy of estimation, Figs. 6-9 depict the RMSE and the minimal upper bound for four nodes respectively, it is easy to see that the RMSE of each node always stays below its minimal upper bound. Overall, the above simulation results verify the feasibility and effectiveness of the developed estimator in this paper.

\section{CONCLUSION}

In this paper, the recursive state estimation problem has been tackled for a class of discrete-time stochastic complex networks with packet disorders under Round-Robin (RR) communication protocols. We have considered the packet disorder issue caused by the random transmission delays that have been modelled by a set of random variables satisfying certain known probability distributions. In order to save the
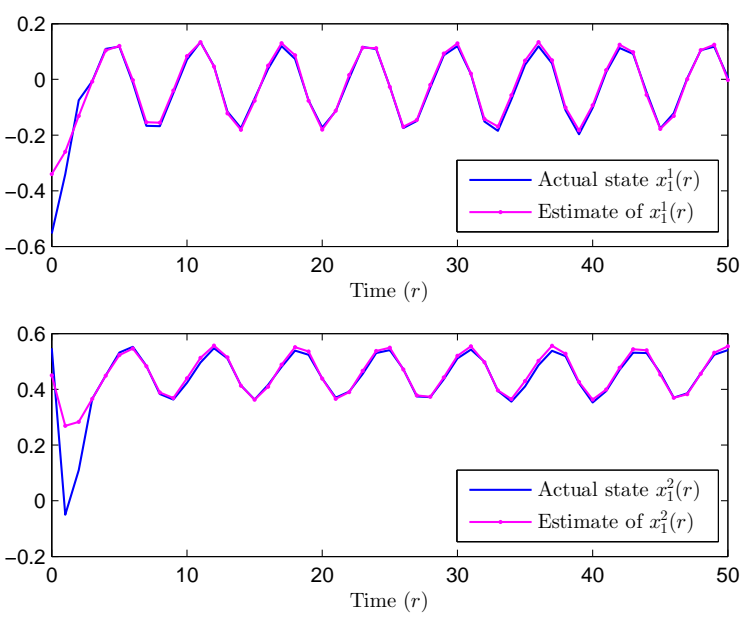

Fig. 2. The actual states $x_{1}^{1}(r), x_{1}^{2}(r)$ and their estimates $\hat{x}_{1}^{1}(r), \hat{x}_{1}^{2}(r)$ of node 1 .
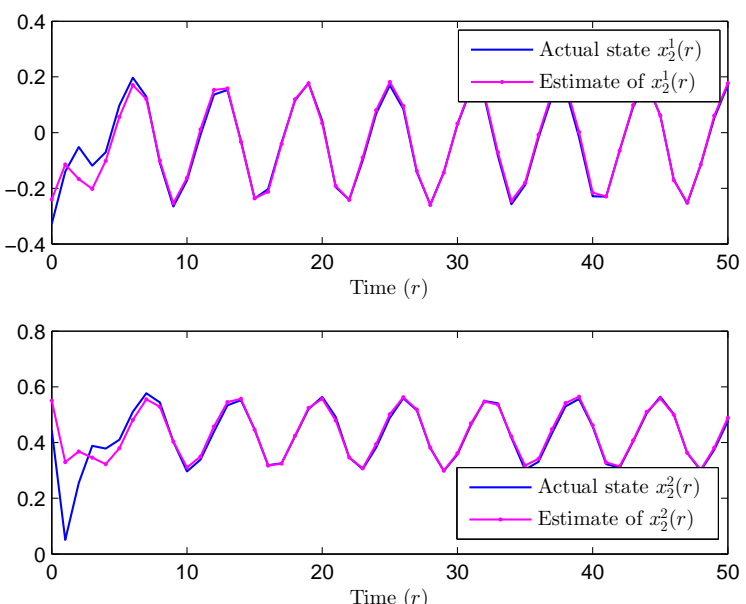

Fig. 3. The actual states $x_{2}^{1}(r), x_{2}^{2}(r)$ and their estimates $\hat{x}_{2}^{1}(r), \hat{x}_{2}^{2}(r)$ of node 2 .

constrained communication resource and also reduce the data collisions, we have introduced the RR protocol that acts as a governor of the ordering of the network nodes gaining the right for data transmission. A recursive estimator has been devised with guaranteed upper bound for the estimation error covariance, and such an upper bound has then been minimized by adequately designing the estimator parameters. Furthermore, we have discussed the boundedness of estimation in the sense of mean square with the help of stochastic analysis techniques. Finally, we have utilized a simulation example to demonstrate the usefulness of the developed estimator design scheme. The possible topics for the future research would be the extension of the main results in this paper to the filtering problems for more complex systems subject to various communication protocols. 
This article has been accepted for publication in a future issue of this journal, but has not been fully edited. Content may change prior to final publication Citation information: DOI10.1109/TNSE.2021.3095217, IEEE Transactions on Network Science and Engineering
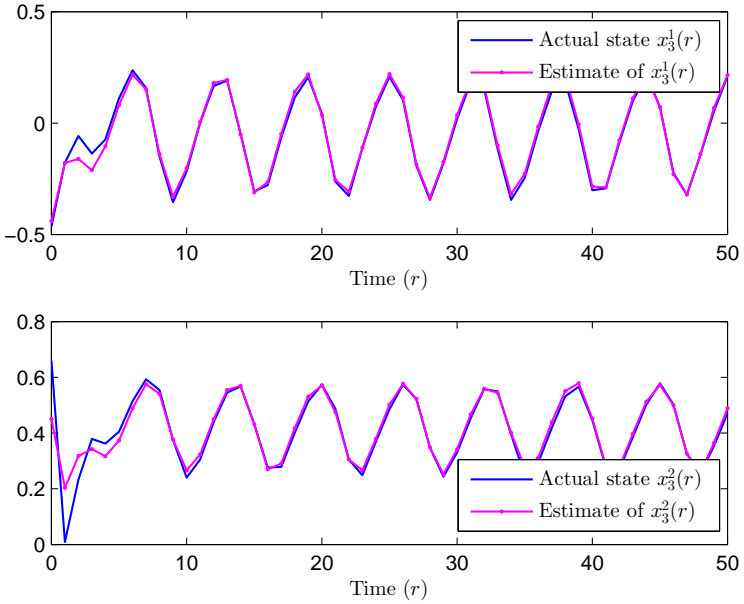

Fig. 4. The actual states $x_{3}^{1}(r), x_{3}^{2}(r)$ and their estimates $\hat{x}_{3}^{1}(r), \hat{x}_{3}^{2}(r)$ of node 3 .
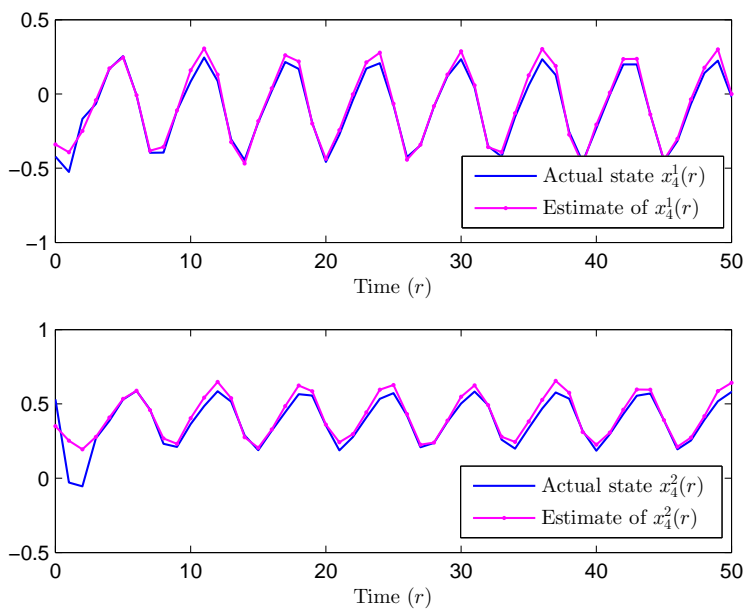

Fig. 5. The actual states $x_{4}^{1}(r), x_{4}^{2}(r)$ and their estimates $\hat{x}_{4}^{1}(r), \hat{x}_{4}^{2}(r)$ of node 4 .

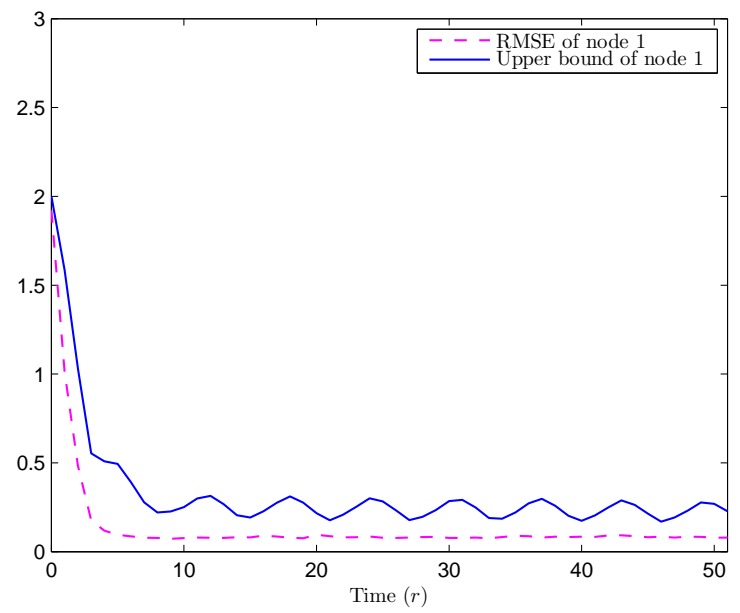

Fig. 6. RMSE and its upper bound of node 1.

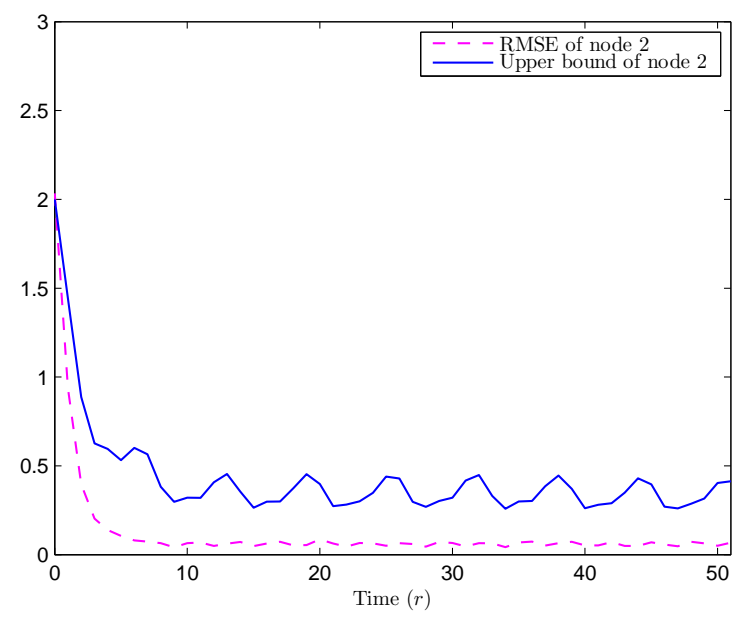

Fig. 7. RMSE and its upper bound of node 2 .

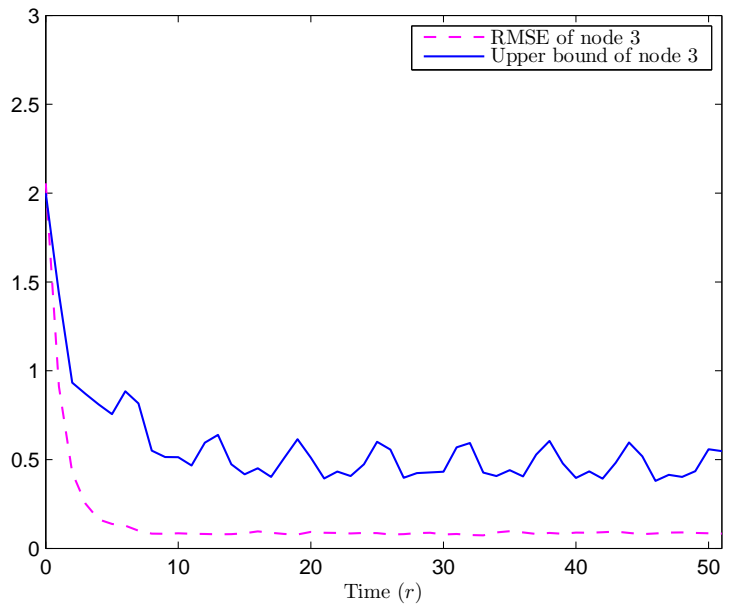

Fig. 8. RMSE and its upper bound of node 3.

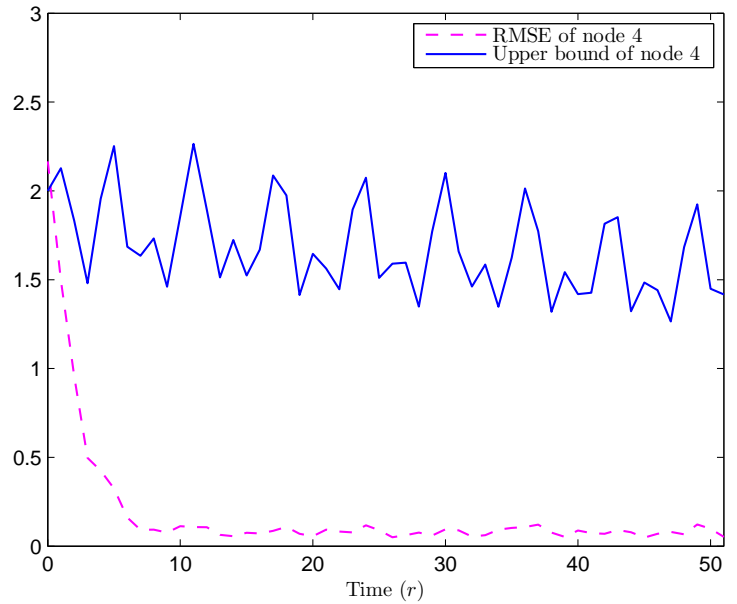

Fig. 9. RMSE and its upper bound of node 4.

\section{REFERENCES}

[1] R. Albert, H. Jeong and A.-L. Barabási, Internet: Diameter of the worldwide web, Nature, vol. 401, no. 9, pp. 130-131, Sept. 1999. 
[2] R. Albert and A.-L. Barabási, Statistical mechanics of complex networks, Reviews of Modern Physics, vol. 74, no. 1, pp. 47-97, Jan. 2002

[3] A. Arenas, A. Díaz-Guilera, J. Kurths, Y. Moreno and C. Zhou, Synchronization in complex networks, Physics Reports, vol. 469, no. 3, pp. 93-153, Dec. 2008.

[4] S. Boccaletti, V. Latora, Y. Moreno, M. Chavez and D.-U. Hwang, Complex networks: Structure and dynamics, Physics Reports, vol. 424, no. 4-5, pp. 175-308, Feb. 2006.

[5] R. Caballero-Águila, A. Hermoso-Carazo and J. Linares-Pérez, Networked fusion estimation with multiple uncertainties and time-correlated channel noise, Information Fusion, vol. 54, pp. 161-171, 2020.

[6] H. Chen, J. Gao, T. Shi and R. Lu, $H_{\infty}$ control for networked control systems with time delay, data packet dropout and disorder, Neurocomputing, vol. 179, pp. 211-218, Feb. 2016.

[7] D. Ding, Z. Wang and Q.-L. Han, Neural-network-based output-feedback control with stochastic communication protocols, Automatica, vol. 106, pp. 221-229, 2019.

[8] N. Eagle and A. S. Pentland, Reality mining: sensing complex social systems, Personal and Ubiquitous Computing, vol. 10, no. 4, pp. 225268, May 2006.

[9] T. Emmerich, A. Bunde and S. Havlin, Diffusion, annihilation, and chemical reactions in complex networks with spatial constraints, Physical Review E, vol. 86, no. 4, pp. 046103-1-046103-5, Oct. 2012.

[10] D. A. Haughton and G. T. Heydt, A linear state estimation formulation for smart distribution systems, IEEE Transactions on Power Systems, vol. 28, no. 2, pp. 1187-1195, May 2013.

[11] N. Hou, Z. Wang, D. W. C. Ho and H. Dong, Robust partial-nodes-based state estimation for complex networks under deception attacks, IEEE Transactions on Cybernetics, vol. 50, no. 6, pp. 2793-2802, 2020.

[12] J. Hu, Z. Wang, G.-P. Liu and H. Zhang, Variance-constrained recursive state estimation for time-varying complex networks with quantized measurements and uncertain inner coupling, IEEE Transactions on Neural Networks and Learning Systems, vol. 31, no. 6, pp. 1955-1967, Jun 2020.

[13] J. Hu, Z. Wang, S. Liu and H. Gao, A variance-constrained approach to recursive state estimation for time-varying complex networks with missing measurements, Automatica, vol. 64, pp. 155-162, Feb. 2016.

[14] J. Hu, H. Zhang, H. Liu and X. Yu, A survey on sliding mode control for networked control systems, International Journal of Systems Science, vol. 52, no. 6, pp. 1129-1147, 2021.

[15] J. Li, Z. Wang, H. Dong and F. Han, Delay-distribution-dependent state estimation for neural networks under stochastic communication protocol with uncertain transition probabilities, Neural Networks, vol. 130 pp. 143-151, 2020.

[16] Q. Li, Z. Wang, W. Sheng, F. E. Alsaadi and F. E. Alsaadi, Dynamic event-triggered mechanism for $H_{\infty}$ non-Fragile state estimation of complex networks under randomly occurring sensor saturations, Information Sciences, vol. 509, pp. 304-316, 2020.

[17] X. Li, F. Han, N. Hou, H. Dong and H. Liu, Set-membership filtering for piecewise linear systems with censored measurements under RoundRobin protocol, International Journal of Systems Science, vol. 51, no. 9, pp. $1578-1588,2020$

[18] A. Liu, W.-A. Zhang, B. Chen and L. Yu, Networked filtering with Markov transmission delays and packet disordering, IET Control Theory \& Applications, vol. 12, no. 5, pp. 687-693, Mar. 2018.

[19] A. Liu, W.-A. Zhang, L. Yu, S. Liu and M. Z. Q. Chen, New results on stabilization of networked control systems with packet disordering, Automatica, vol. 52, pp. 255-259, Feb. 2015.

[20] D. Liu, Y. Liu and F. E. Alsaadi, Recursive state estimation based-on the outputs of partial nodes for discrete-time stochastic complex networks with switched topology, Journal of the Franklin Institute, vol. 355, no. 11, pp. 4686-4707, Jul. 2018.

[21] D. Liu, Z. Wang, Y. Liu and F. E. Alsaadi, Extended Kalman filtering subject to random transmission delays: Dealing with packet disorders, Information Fusion, vol. 60, pp. 80-86, Aug. 2020.

[22] L. Liu, L. Ma, J. Zhang and Y. Bo, Distributed non-fragile setmembership filtering for nonlinear systems under fading channels and bias injection attacks, International Journal of Systems Science, vol. 52, no. 6, pp. 1192-1205, 2021.

[23] S. Liu, Z. Wang, Y. Chen and G. Wei, Protocol-based unscented Kalman filtering in the presence of stochastic uncertainties, IEEE Transactions on Automatic Control, vol. 65, no. 3, pp. 1303-1309, Mar. 2020.

[24] S. Liu, Z. Wang, G. Wei and M. Li, Distributed set-membership filtering for multirate systems under the Round-Robin scheduling over sensor networks, IEEE Transactions on Cybernetics, vol. 50, no. 5, pp. 1910 1920, May 2020.
[25] Y. Liu, Z. Wang, L. Ma and F. E. Alsaadi, A partial-nodes-based information fusion approach to state estimation for discrete-time delayed stochastic complex networks, Information Fusion, vol. 49, pp. 240-248, Sept. 2019.

[26] J. Mao, D. Ding, G. Wei and H. Liu, Networked recursive filtering for time-delayed nonlinear stochastic systems with uniform quantisation under Round-Robin protocol, International Journal of Systems Science, vol. 50, no. 4, pp. 871-884, Mar. 2019.

[27] C. Peng, Y.-C. Tian and D. Yue, Output feedback control of discretetime systems in networked environments, IEEE Transactions on Systems, Man, and Cybernetics-Part A: Systems and Humans, vol. 41, no. 1, pp. 185-190, 2010.

[28] K. Reif, S. Günther, E. Yaz and R. Unbehauen, Stochastic stability of the discrete-time extended Kalman filter, IEEE Transactions on Automatic Control, vol. 44, no. 4, pp. 714-728, Apr. 1999.

[29] B. Shen, Z. Wang and X. Liu, Bounded $H_{\infty}$ synchronization and state estimation for discrete time-varying stochastic complex for discrete time-varying stochastic complex networks over a finite horizon, IEEE Transactions on Neural Networks, vol. 22, no. 1, pp. 145-157, Jan. 2011.

[30] Y. Shen, Z. Wang, B. Shen, F. E. Alsaadi and F. E. Alsaadi, Fusion estimation for multi-rate linear repetitive processes under weighted Try-Once-Discard protocol, Information Fusion, vol. 55, pp. 281-291, Mar. 2020.

[31] J. Song, Z. Wang and Y. Niu, On $H_{\infty}$ sliding mode control under stochastic communication protocol, IEEE Transactions on Automatic Control, vol. 64, no. 5, pp. 2174-2181, 2019.

[32] M. Tabbara and D. Nesic, Input-output stability of networked control systems with stochastic protocols and channels, IEEE Transactions on Automatic Control, vol. 53, no. 5, pp. 1160-1175, Jun. 2008.

[33] E. Tian, D. Yue and C. Peng, Reliable control for networked control systems with probabilistic actuator fault and random delays, Journal of the Franklin Institute, vol. 347, no. 10, pp. 1907-1926, 2010.

[34] F. Wang, Z. Wang, J. Liang and X. Liu, Robust finite-horizon filtering for 2-D systems with randomly varying sensor delays, IEEE Transactions on Systems, Man, and Cybernetics - Systems, vol. 50, no. 1, pp. 220-232, Jan. 2020.

[35] Z. Wang, Y. Wang and Y. Liu, Global synchronization for discrete-time stochastic complex networks with randomly occurred nonlinearities and mixed time-delays, IEEE Transactions on Neural Networks, vol. 21, no. 1, pp. 11-25, Jan. 2010.

[36] L. Xie, Y. C. Soh and C. E. de Souza, Robust Kalman filtering for uncertain discrete-time systems, IEEE Transactions on Automatic Control, vol. 39, no. 6, pp. 1310-1314, Jun. 1994.

[37] Y. Xu, R. Lu, P. Shi, H. Li and S. Xie, Finite-time distributed state estimation over sensor networks with Round-Robin protocol and fading channels, IEEE Transactions on Cybernetics, vol. 48, no. 1, pp. 336345, Jan. 2018.

[38] Y. Xu, R. Lu, P. Shi, H. Li and S. Xie, Finite-time distributed state estimation over sensor networks with Round-Robin protocol and fading channels, IEEE Transactions on Cybernetics, vol. 48, no. 1, pp. 336-345, Jan. 2018.

[39] W. Xu, G. Hu, D. W. C. Ho and Z. Feng, Distributed secure cooperative control under denial-of-service attacks from multiple adversaries, IEEE Transactions on Cybernetics, vol. 50, no. 8, pp. 3458-3467, 2020.

[40] W. Yu, G. Chen and J. Cao, Adaptive synchronization of uncertain coupled stochastic complex networks, Asian Journal of Control, vol. 13, no. 3, pp. 418-429, Apr. 2011.

[41] Y.-B. Zhao, G.-P. Liu and D. Rees, Design of a packet-based control framework for networked control systems, IEEE Transactions on Control Systems Technology, vol. 17, no. 4, pp. 859-865, Jul. 2009.

[42] Y.-B. Zhao, G.-P. Liu and D. Rees, Actively compensating for data packet disorder in networked control systems, IEEE Transactions on Circuits and Systems II: Express Briefs, vol. 57, no. 11, pp. 913-917, Nov. 2010.

[43] Z. Zhao, Z. Wang, L. Zou and J. Guo, Set-membership filtering for timevarying complex networks with uniform quantisations over randomly delayed redundant channels, International Journal of Systems Science, vol. 51, no. 16, pp. 3364-3377, 2020.

[44] K. Zhu, J. Hu, Y. Liu, N. D. Alotaibi and F. E. Alsaadi, On $\ell_{2}-\ell_{\infty}$ output-feedback control scheduled by stochastic communication protocol for two-dimensional switched systems, International Journal of Systems Science, in press, DOI: 10.1080/00207721.2021.1914768.

[45] L. Zou, Z. Wang and H. Gao, Observer-based $H_{\infty}$ control of networked systems with stochastic communication protocol: The finite-horizon case, Automatica, vol. 63, pp. 366-373, Jan. 2016.

[46] L. Zou, Z. Wang, H. Gao and X. Liu, State estimation for discretetime dynamical networks with time-varying delays and stochastic 
disturbances under the Round-Robin protocol, IEEE Transactions on Neural Networks and Learning Systems, vol. 28, no. 5, pp. 1139-1151, May 2017.

[47] L. Zou, Z. Wang, J. Hu, Y. Liu and X. Liu, Communication-protocolbased analysis and synthesis of networked systems: Progress, prospects and challenges, International Journal of Systems Science, in press, DOI 10.1080/00207721.2021.1917721.

[48] L. Zou, Z. Wang, Q.-L. Han and D. H. Zhou, Moving horizon estimation of networked nonlinear systems with random access protocol, IEEE Transactions on Systems, Man, and Cybernetics: Systems, vol. 51, no. 5, pp. 2937-2948, 2021

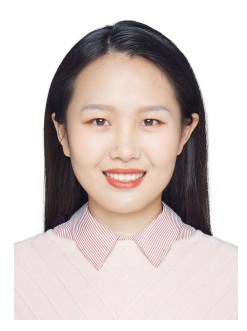

Dan Liu received the BSc degree in Mathematics and Applied Mathematics from Yangzhou University, Yangzhou, China, in 2014, and the PhD degree in Applied Mathematics from Yangzhou University, Yangzhou, China, in 2020.

Dr. Liu is currently a lecturer in the Department of Mathematics at Yangzhou University, China. Her current research interests are Control and filtering of complex networks.

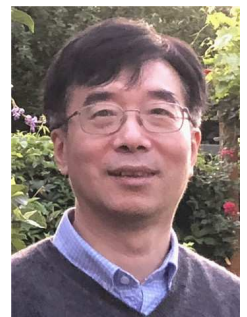

Zidong Wang (SM'03-F'14) was born in Jiangsu, China, in 1966. He received the B.Sc. degree in mathematics in 1986 from Suzhou University, Suzhou, China, and the M.Sc. degree in applied mathematics in 1990 and the Ph.D. degree in electrical engineering in 1994, both from Nanjing University of Science and Technology, Nanjing, China.

$\mathrm{He}$ is currently Professor of Dynamical Systems and Computing in the Department of Computer Science, Brunel University London, U.K. From 1990 to 2002, he held teaching and research appointments in universities in China, Germany and the UK. Prof. Wang's research interests include dynamical systems, signal processing, bioinformatics, control theory and applications. He has published more than 600 papers in international journals. He is a holder of the Alexander von Humboldt Research Fellowship of Germany, the JSPS Research Fellowship of Japan, William Mong Visiting Research Fellowship of Hong Kong.

Prof. Wang serves (or has served) as the Editor-in-Chief for International Journal of Systems Science, the Editor-in-Chief for Neurocomputing, the Editor-in-Chief for Systems Science \& Control Engineering, and an Associate Editor for 12 international journals including IEEE Transactions on Automatic Control, IEEE Transactions on Control Systems Technology, IEEE Transactions on Neural Networks, IEEE Transactions on Signal Processing, and IEEE Transactions on Systems, Man, and Cybernetics-Part C. He is a Member of the Academia Europaea, a Member of the European Academy of Sciences and Arts, an Academician of the International Academy for Systems and Cybernetic Sciences, a Fellow of the IEEE, a Fellow of the Royal Statistical Society and a member of program committee for many international conferences.

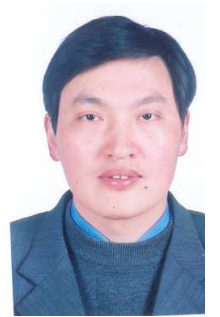

Yurong Liu received his B.Sc. degree in Mathematics from Suzhou University, Suzhou, China, in 1986, the M.Sc. degree in Applied Mathematics from Nanjing University of Science and Technology, Nanjing, China, in 1989, and the Ph.D. degree in Applied Mathematics from Suzhou University, Suzhou, China, in 2000.

Dr. Liu is now a Professor at the Department of Mathematics, Yangzhou University, China. His current interests include neural networks, nonlinear dynamics, time-delay systems, and chaotic dynamics. He has published more than 100 papers in international journal and serves as an editorial board member for Neurocomputing.

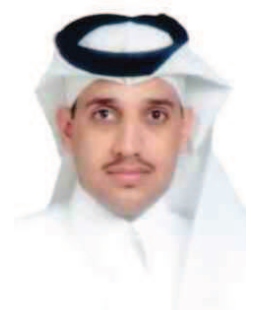

Fawaz E. Alsaadi received the B.S. and M.Sc. degrees in computer science from King Abdulaziz University, Jeddah, Saudi Arabia, and University of Denver, Denver, Colorado, USA respectively. $\mathrm{He}$ then received the Ph.D. degree in biometric security from the University of Colorado Springs, Colorado Springs, USA. He is currently an associate professor in the Information Technology Department within the Faculty of Computing and Information Technology, King Abdulaziz University, Jeddah, Saudi Arabia. He has research interests in biometric recognition and biometric security, information security, and cloud computing storage security.

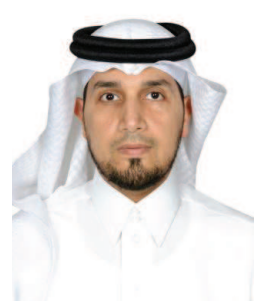

Fuad E. Alsaadi received the B.S. and M.Sc. degrees in electronic and communication from King AbdulAziz University, Jeddah, Saudi Arabia, in 1996 and 2002, respectively and the Ph.D. degree in optical wireless communication systems from the University of Leeds, Leeds, U.K., in 2011. Between 1996 and 2005, he worked in Jeddah as a Communication Instructor with the College of Electronics \& Communication. He is currently an Associate Professor of the Electrical and Computer Engineering Department within the Faculty of Engineering, King Abdulaziz University, Jeddah, Saudi Arabia. He has published widely in the top IEEE Communications Conferences and Journals. His research interests include optical systems and networks, signal processing, synchronization and systems design. He was the recipient of the Carter Award, University of Leeds for the best Ph.D. 